Document downloaded from:

http://hdl.handle.net/10251/52827

This paper must be cited as:

Benítez López, J.; Sarduvan, M.; Ülker, S.; Özdemir, H. (2013). On nonsingularity of combinations of three group invertible matrices and three tripotent matrices. Linear and Multilinear Algebra. 61(4):463-481. doi:10.1080/03081087.2012.689986.

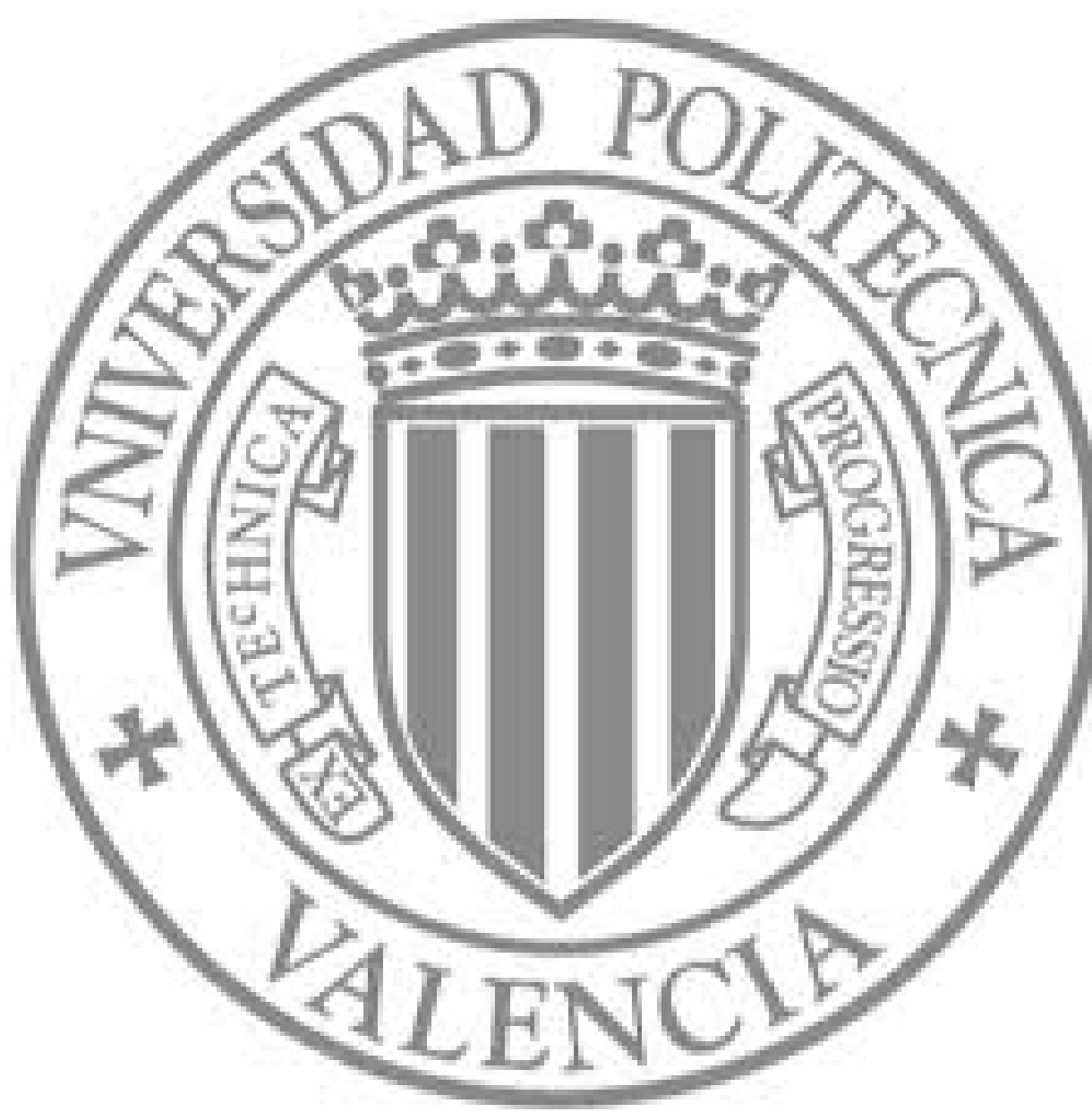

The final publication is available at

http://dx.doi.org/10.1080/03081087.2012.689986

Copyright Taylor \& Francis 


\title{
On nonsingularity of combinations of three group invertible matrices and three tripotent matrices
}

\begin{abstract}
Let $\mathbf{T}=c_{1} \mathbf{T}_{1}+c_{2} \mathbf{T}_{2}+c_{3} \mathbf{T}_{3}-c_{4}\left(\mathbf{T}_{1} \mathbf{T}_{2}+\mathbf{T}_{3} \mathbf{T}_{1}+\mathbf{T}_{2} \mathbf{T}_{3}\right)$, where $\mathbf{T}_{1}, \mathbf{T}_{2}, \mathbf{T}_{3}$ are three $n \times n$ tripotent matrices and $c_{1}, c_{2}, c_{3}, c_{4}$ are complex numbers with $c_{1}, c_{2}, c_{3}$ nonzero. In this paper, it is mainly established necessary and sufficient conditions for the nonsingularity of such combinations and obtained some formulae for the inverses of them. Some of these results are given in terms of group invertible matrices.
\end{abstract}

AMS classification: 15A18; 15B99; 15A09

Keywords: Nonsingularity; Tripotent matrix; Group invertible matrix; Combination; Diagonalization

\section{Introduction and Preliminaries}

Let $\mathbb{C}$ be the field of complex numbers and $\mathbb{C}^{*}=\mathbb{C} \backslash\{0\}$. For a positive integer $n$, let $\mathcal{M}_{n}$ be the set of all $n \times n$ complex matrices over $\mathbb{C}$. The symbols $\operatorname{rank}(\mathbf{A}), \mathbf{A}^{*}, \mathcal{R}(\mathbf{A})$, and $\mathcal{N}(\mathbf{A})$ stands for the rank, conjugate transpose, the range space, and the null space of $\mathbf{A} \in \mathcal{M}_{n}$, respectively. Recall that a matrix $\mathbf{A} \in \mathcal{M}_{n}$ is idempotent if $\mathbf{A}^{2}=\mathbf{A}$ and tripotent if $\mathbf{A}^{3}=\mathbf{A}$.

The nonsingularity of linear combinations of idempotent matrices and $k$-potent matrices was studied in, for example, $[1,2,4,6,9,15]$. The nonsingularities of the combinations $c_{1} \mathbf{P}+c_{2} \mathbf{Q}-c_{3} \mathbf{P Q}$ and $c_{1} \mathbf{P}+c_{2} \mathbf{Q}-c_{3} \mathbf{P Q}-c_{4} \mathbf{Q P}-c_{5} \mathbf{P Q P}$ of two idempotent matrices $\mathbf{P}, \mathbf{Q}$ were investigated in [16] and [17], respectively. The considerations of this paper are inspired by Liu et al.[10]. They established necessary and sufficient conditions for the nonsingularity of combinations $c_{1} \mathbf{T}_{1}+c_{2} \mathbf{T}_{2}-c_{3} \mathbf{T}_{1} \mathbf{T}_{2}$ of two trioptent matrices and gave some formulae for the inverse of $c_{1} \mathbf{T}_{1}+c_{2} \mathbf{T}_{2}-c_{3} \mathbf{T}_{1} \mathbf{T}_{2}$ under the some conditions.

Consider a combination of the form

$$
\mathbf{T}=c_{1} \mathbf{T}_{1}+c_{2} \mathbf{T}_{2}+c_{3} \mathbf{T}_{3}-c_{4}\left(\mathbf{T}_{1} \mathbf{T}_{2}+\mathbf{T}_{3} \mathbf{T}_{1}+\mathbf{T}_{2} \mathbf{T}_{3}\right)
$$

where $c_{1}, c_{2}, c_{3} \in \mathbb{C}^{*}, c_{4} \in \mathbb{C}$ and $\mathbf{T}_{1}, \mathbf{T}_{2}, \mathbf{T}_{3} \in \mathcal{M}_{n}$ are three tripotent matrices. The purpose of this paper is mainly twofold: first, to establish necessary and sufficient conditions for the nonsingularity of combinations of the form (1.1) and then to give some formulae for the inverse of them.

Now, let us give the following additional concepts and properties. For a given matrix $\mathbf{A} \in \mathcal{M}_{n}$ is said to be group invertible if there exists a matrix $\mathbf{X} \in \mathcal{M}_{n}$ such that

$$
\mathbf{A X A}=\mathbf{A}, \quad \mathbf{X A X}=\mathbf{X}, \quad \mathbf{A X}=\mathbf{X A}
$$

hold. If such an $\mathbf{X} \in \mathcal{M}_{n}$ exists, then it is unique, customarily denoted by $\mathbf{A}^{\#}$ [3]. A matrix $\mathbf{A} \in \mathcal{M}_{n}$ is group invertible if and only if there exist nonsingular $\mathbf{S} \in \mathcal{M}_{n}, \mathbf{C} \in \mathcal{M}_{r}$ such that $\mathbf{A}=\mathbf{S}(\mathbf{C} \oplus \mathbf{0}) \mathbf{S}^{-1}, r$ being the rank of $\mathbf{A}$ [12, Exercise 5.10.12]. In this situation, one has $\mathbf{A}^{\#}=\mathbf{S}\left(\mathbf{C}^{-1} \oplus \mathbf{0}\right) \mathbf{S}^{-1}$. This latter representation implies that any diagonalizable 
matrix is group invertible. Moreover, it is well known that $\mathbf{A} \in \mathcal{M}_{n}$ is nonsingular if and only if $\mathcal{N}(\mathbf{A})=\{\mathbf{0}\}$. Furthermore, if $\mathbf{A} \in \mathcal{M}_{n}$ and $k$ is a natural number greater than 1 , then $\mathbf{A}$ satisfies $\mathbf{A}^{k}=\mathbf{A}$ if and only if $\mathbf{A}$ is diagonalizable and the spectrum of $\mathbf{A}$ is contained in $\sqrt[k-1]{1} \cup\{0\}[5]$.

Special types of matrices, such as idempotents, tripotents, etc., are very useful in many contexts and they have been extensively studied in the literature. For example, quadratic forms with idempotent matrices are used extensively in statistical theory. So it is worth to stress and spread these kinds of results. Evidently, if $\mathbf{T}$ is a tripotent matrix, then $\mathbf{T}$ is group invertible and $\mathbf{T}^{\#}=\mathbf{T}$. Many of the results given in this work will be given in terms of group invertible matrices.

\section{Main Results}

Baksalary and Baksalary [1] proved that the nonsingularity of $\mathbf{P}_{1}+\mathbf{P}_{2}$, where $\mathbf{P}_{1}$ and $\mathbf{P}_{2}$ are idempotent matrices, is equivalent to the nonsingularity of any linear combinations $c_{1} \mathbf{P}_{1}+c_{2} \mathbf{P}_{2}$, where $c_{1}, c_{2} \in \mathbb{C} \backslash\{0\}$ and $c_{1}+c_{2} \neq 0$. This result was further generalized in [8], where it was proved the stability of the nullity and rank of $c_{1} \mathbf{P}_{1}+c_{2} \mathbf{P}_{2}$ for any $c_{1}, c_{2} \in \mathbb{C} \backslash\{0\}$. In the forthcoming results, we give similar results for two and three commuting tripotent matrices. For another related paper concerning this topic, the reader is referred to [15]. We need the following simple lemma whose proof is left to the reader

Lemma 2.1. Let $\mathbf{A}, \mathbf{B} \in \mathcal{M}_{n}$ be two group invertible matrices such that there exist nonsingular matrices $\mathbf{S} \in \mathcal{M}_{n}, \mathbf{A}_{1}, \mathbf{B}_{1} \in \mathcal{M}_{r}$ satisfying $\mathbf{A}=\mathbf{S}\left(\mathbf{A}_{1} \oplus \mathbf{0}\right) \mathbf{S}^{-1}$ and $\mathbf{B}=\mathbf{S}\left(\mathbf{B}_{1} \oplus \mathbf{0}\right) \mathbf{S}^{-1}$. Then $\mathcal{R}(\mathbf{A})=\mathcal{R}(\mathbf{B})$ and $\mathcal{N}(\mathbf{A})=\mathcal{N}(\mathbf{B})$.

Theorem 2.1. Let $\mathbf{T}_{1}, \mathbf{T}_{2} \in \mathcal{M}_{n} \backslash\{\mathbf{0}\}$ be two commuting tripotent matrices and $c_{1}, c_{2} \in \mathbb{C}^{*}$ such that $c_{1}^{2}-c_{2}^{2} \neq 0$. Then $\mathcal{R}\left(\mathbf{T}_{1}^{2}+\mathbf{T}_{2}^{2}\right)=\mathcal{R}\left(c_{1} \mathbf{T}_{1}+c_{2} \mathbf{T}_{2}\right), \mathcal{N}\left(\mathbf{T}_{1}^{2}+\mathbf{T}_{2}^{2}\right)=\mathcal{N}\left(c_{1} \mathbf{T}_{1}+c_{2} \mathbf{T}_{2}\right)$, $c_{1} \mathbf{T}_{1}+c_{2} \mathbf{T}_{2}$ is group invertible and

$$
\left(c_{1} \mathbf{T}_{1}+c_{2} \mathbf{T}_{2}\right)^{\#}=\frac{c_{2}^{2}}{c_{1}\left(c_{1}^{2}-c_{2}^{2}\right)} \mathbf{T}_{1} \mathbf{T}_{2}^{2}+\frac{c_{1}^{2}}{c_{2}\left(c_{2}^{2}-c_{1}^{2}\right)} \mathbf{T}_{2} \mathbf{T}_{1}^{2}+\frac{1}{c_{1}} \mathbf{T}_{1}+\frac{1}{c_{2}} \mathbf{T}_{2}
$$

In particular, If $\mathbf{T}_{1}^{2}+\mathbf{T}_{2}^{2}$ is nonsingular, then $c_{1} \mathbf{T}_{1}+c_{2} \mathbf{T}_{2}$ is nonsingular and $\left(c_{1} \mathbf{T}_{1}+c_{2} \mathbf{T}_{2}\right)^{-1}$ is given by (2.1).

Proof. Let $p=\operatorname{rank}\left(\mathbf{T}_{1} \mathbf{T}_{2}\right), q=\operatorname{rank}\left(\mathbf{T}_{1}\right)$, and $r=\operatorname{rank}\left(\mathbf{T}_{2}\right)$. Since $\mathbf{T}_{1}$ and $\mathbf{T}_{2}$ are diagonalizable and commuting, there exists a nonsingular $\mathbf{S} \in \mathcal{M}_{n}$ such that

$$
\mathbf{T}_{1}=\mathbf{S}\left(\mathbf{A}_{1} \oplus \mathbf{B}_{1} \oplus \mathbf{0} \oplus \mathbf{0}\right) \mathbf{S}^{-1}, \quad \mathbf{T}_{2}=\mathbf{S}\left(\mathbf{A}_{2} \oplus \mathbf{0} \oplus \mathbf{B}_{2} \oplus \mathbf{0}\right) \mathbf{S}^{-1},
$$

being $\mathbf{A}_{1}, \mathbf{A}_{2} \in \mathcal{M}_{p}, \mathbf{B}_{1}=\mathcal{M}_{q-p}, \mathbf{B}_{2} \in \mathcal{M}_{r-p}$, and $\mathbf{A}_{1}, \mathbf{A}_{2}, \mathbf{B}_{1}, \mathbf{B}_{2}$ nonsingular. By using $\mathbf{T}_{1}^{3}=\mathbf{T}_{1}$ and $\mathbf{T}_{2}^{3}=\mathbf{T}_{2}$ one gets $\mathbf{A}_{1}^{2}=\mathbf{A}_{2}^{2}=\mathbf{I}_{p}, \mathbf{B}_{1}^{2}=\mathbf{I}_{q-p}$, and $\mathbf{B}_{2}^{2}=\mathbf{I}_{r-p}$. Therefore,

$$
\mathbf{T}_{1}^{2}+\mathbf{T}_{2}^{2}=\mathbf{S}\left(2 \mathbf{I}_{p} \oplus \mathbf{I}_{q-p} \oplus \mathbf{I}_{r-p} \oplus \mathbf{0}\right) \mathbf{S}^{-1}
$$

64 By considering the equality

$$
\left(c_{1} \mathbf{A}_{1}+c_{2} \mathbf{A}_{2}\right)\left(c_{1} \mathbf{A}_{1}-c_{2} \mathbf{A}_{2}\right)=\left(c_{1}^{2}-c_{2}^{2}\right) \mathbf{I}_{p}
$$

${ }_{5}$ we get the nonsingularity of $c_{1} \mathbf{A}_{1}+c_{2} \mathbf{A}_{2}$. Since

$$
c_{1} \mathbf{T}_{1}+c_{2} \mathbf{T}_{2}=\mathbf{S}\left[\left(c_{1} \mathbf{A}_{1}+c_{2} \mathbf{A}_{2}\right) \oplus c_{1} \mathbf{B}_{1} \oplus c_{2} \mathbf{B}_{2} \oplus \mathbf{0}\right] \mathbf{S}^{-1},
$$

and by applying Lemma 2.1 to matrices $\mathbf{T}_{1}^{2}+\mathbf{T}_{2}^{2}$ and $c_{1} \mathbf{T}_{1}+c_{2} \mathbf{T}_{2}$ we obtain the equality of the range spaces and null spaces of this theorem. Also, $\mathbf{B}_{1}^{2}=\mathbf{I}_{q-p}, \mathbf{B}_{2}^{2}=\mathbf{I}_{r-p}$, the expression (2.4), and [12, Exercise 5.10.12] permit assure that $c_{1} \mathbf{T}_{1}+c_{2} \mathbf{T}_{2}$ is group invertible and

$$
\left(c_{1} \mathbf{T}_{1}+c_{2} \mathbf{T}_{2}\right)^{\#}=\mathbf{S}\left[\left(c_{1} \mathbf{A}_{1}+c_{2} \mathbf{A}_{2}\right)^{-1} \oplus c_{1}^{-1} \mathbf{B}_{1} \oplus c_{2}^{-1} \mathbf{B}_{2} \oplus \mathbf{0}\right] \mathbf{S}^{-1}
$$


Now we use the equality (2.3):

$$
\begin{aligned}
{\left[\left(c_{1} \mathbf{A}_{1}+c_{2} \mathbf{A}_{2}\right)^{-1} \oplus \mathbf{0} \oplus \mathbf{0} \oplus \mathbf{0}\right] } & =\frac{1}{c_{1}^{2}-c_{2}^{2}}\left[\left(c_{1} \mathbf{A}_{1}-c_{2} \mathbf{A}_{2}\right) \oplus \mathbf{0} \oplus \mathbf{0} \oplus \mathbf{0}\right] \\
& =\frac{1}{c_{1}^{2}-c_{2}^{2}}\left[c_{1}\left(\mathbf{A}_{1} \oplus \mathbf{0} \oplus \mathbf{0} \oplus \mathbf{0}\right)-c_{2}\left(\mathbf{A}_{2} \oplus \mathbf{0} \oplus \mathbf{0} \oplus \mathbf{0}\right)\right] \\
& =\frac{1}{c_{1}^{2}-c_{2}^{2}}\left[c_{1} \mathbf{S}^{-1} \mathbf{T}_{1} \mathbf{T}_{2}^{2} \mathbf{S}-c_{2} \mathbf{S}^{-1} \mathbf{T}_{1}^{2} \mathbf{T}_{2} \mathbf{S}\right]
\end{aligned}
$$

In addition we have $\mathbf{S}\left(\mathbf{0} \oplus \mathbf{B}_{1} \oplus \mathbf{0} \oplus \mathbf{0}\right) \mathbf{S}^{-1}=\mathbf{T}_{1}\left(\mathbf{I}_{n}-\mathbf{T}_{2}^{2}\right)$ and $\mathbf{S}\left(\mathbf{0} \oplus \mathbf{0} \oplus \mathbf{B}_{2} \oplus \mathbf{0}\right) \mathbf{S}^{-1}=$ $\mathbf{T}_{2}\left(\mathbf{I}_{n}-\mathbf{T}_{1}^{2}\right)$. Therefore

$$
\left(c_{1} \mathbf{T}_{1}+c_{2} \mathbf{T}_{2}\right)^{\#}=\frac{1}{c_{1}^{2}-c_{2}^{2}}\left[c_{1} \mathbf{T}_{1} \mathbf{T}_{2}^{2}-c_{2} \mathbf{T}_{1}^{2} \mathbf{T}_{2}\right]+\frac{1}{c_{1}} \mathbf{T}_{1}\left(\mathbf{I}_{n}-\mathbf{T}_{2}^{2}\right)+\frac{1}{c_{2}} \mathbf{T}_{2}\left(\mathbf{I}_{n}-\mathbf{T}_{1}^{2}\right) .
$$

Corollary 2.1. Let $\mathbf{T}_{1}, \mathbf{T}_{2} \in \mathcal{M}_{n} \backslash\{\mathbf{0}\}$ be two commuting tripotent matrices satisfying $\mathbf{T}_{1} \mathbf{T}_{2}=\mathbf{0}$ and let $c_{1}, c_{2} \in \mathbb{C}^{*}$. Then $c_{1} \mathbf{T}_{1}+c_{2} \mathbf{T}_{2}$ is group invertible and

$$
\left(c_{1} \mathbf{T}_{1}+c_{2} \mathbf{T}_{2}\right)^{\#}=\frac{1}{c_{1}} \mathbf{T}_{1}+\frac{1}{c_{2}} \mathbf{T}_{2} .
$$

Remark 2.1. Observe that $\mathbf{T}_{1}^{2}+\mathbf{T}_{2}^{2}$ is nonsingular if and only if $\operatorname{rank}\left(\mathbf{T}_{1}\right)+\operatorname{rank}\left(\mathbf{T}_{2}\right)=$ $n+\operatorname{rank}\left(\mathbf{T}_{1} \mathbf{T}_{2}\right)$. In fact, from the representation (2.2) we have

$\mathbf{T}_{1}^{2}+\mathbf{T}_{2}^{2}$ is nonsingular $\Leftrightarrow p+(q-p)+(r-p)=n \Leftrightarrow \operatorname{rank}\left(\mathbf{T}_{1}\right)+\operatorname{rank}\left(\mathbf{T}_{2}\right)=n+\operatorname{rank}\left(\mathbf{T}_{1} \mathbf{T}_{2}\right)$.

The following simple pair of equalities will be useful to prove next result: If $\mathbf{A}$, $\mathbf{B}$, and $\mathbf{C} \in \mathcal{M}_{n}$ satisfy $\mathbf{A}^{2}=\mathbf{B}^{2}=\mathbf{C}^{2}=\mathbf{I}_{n}$ and they are mutually commuting, then

$$
(a \mathbf{A}+b \mathbf{B}+c \mathbf{C})(x \mathbf{A}+y \mathbf{B}+z \mathbf{C}+w \mathbf{A B C})=\left(a^{4}+b^{4}+c^{4}-2 a^{2} b^{2}-2 b^{2} c^{2}-2 c^{2} a^{2}\right) \mathbf{I}_{n},
$$

where $x=a^{3}-a b^{2}-a c^{2}, y=b^{3}-b c^{2}-b a^{2}, z=c^{3}-c a^{2}-c b^{2}, w=2 a b c$, and $a, b, c$ are arbitrary nonzero complex numbers. Furthermore,

$$
a^{4}+b^{4}+c^{4}-2 a^{2} b^{2}-2 b^{2} c^{2}-2 c^{2} a^{2}=(a+b+c)(a+b-c)(a-b+c)(a-b-c)
$$

holds. In addition, the following simple lemma (whose proof is left to the reader) will help us to prove Theorem 2.2 below

Lemma 2.2. Let $\mathbf{B}_{i} \in M_{n_{i}}$ for $i=1, \ldots, m, n=n_{1}+\cdots+n_{m}$, a nonsingular $\mathbf{S} \in M_{n}$. If we define $\mathbf{A}_{i}=\mathbf{S}\left(\mathbf{0} \oplus \cdots \oplus \mathbf{0} \oplus \mathbf{B}_{i} \oplus \mathbf{0} \oplus \cdots \oplus \mathbf{0}\right) \mathbf{S}^{-1}$, where the summand $\mathbf{B}_{i}$ is on the $i t h$ position, and $\mathbf{A}=\mathbf{S}\left(\mathbf{B}_{1} \oplus \cdots \oplus \mathbf{B}_{m}\right) \mathbf{S}^{-1}$, then

$$
\bigcap_{i=1}^{m} \mathcal{N}\left(\mathbf{A}_{i}\right)=\mathcal{N}(\mathbf{A}) \quad \text { and } \quad \sum_{i=1}^{m} \mathcal{R}\left(\mathbf{A}_{i}\right)=\mathcal{R}(\mathbf{A})
$$

In addition, if $\mathbf{B}_{1}, \ldots, \mathbf{B}_{m}$ are group invertible, then $\mathbf{A}$ is also group invertible and $\mathbf{A}^{\#}=$ ${ }_{6} \quad \mathbf{S}\left(\mathbf{B}_{1}^{\#} \oplus \cdots \oplus \mathbf{B}_{m}^{\#}\right) \mathbf{S}^{-1}$.

theo_c77 Theorem 2.2. Let $\mathbf{T}_{1}, \mathbf{T}_{2}, \mathbf{T}_{3} \in \mathcal{M}_{n} \backslash\{\mathbf{0}\}$ be three mutually commuting tripotent matrices and $c_{1}, c_{2}, c_{3} \in \mathbb{C}^{*}$ such that $c_{2}^{2}-c_{3}^{3}, c_{1}^{2}-c_{3}^{2}, c_{1}^{2}-c_{2}^{2}, c_{1}+c_{2}+c_{3}, c_{1}+c_{2}-c_{3}, c_{1}-c_{2}+$ 


$$
\begin{aligned}
\left(c_{1} \mathbf{T}_{1}\right. & \left.+c_{2} \mathbf{T}_{2}+c_{3} \mathbf{T}_{3}\right)^{\#}=q\left(\mathbf{T}_{1}, \mathbf{T}_{2}, \mathbf{T}_{3}\right) \mathbf{T}_{1}^{2} \mathbf{T}_{2}^{2} \mathbf{T}_{3}^{2}+p_{c_{1}, c_{2}}\left(\mathbf{T}_{1}, \mathbf{T}_{2}\right) \mathbf{T}_{1}^{2} \mathbf{T}_{2}^{2}\left(\mathbf{I}_{n}-\mathbf{T}_{3}^{2}\right) \\
& +p_{c_{1}, c_{3}}\left(\mathbf{T}_{1}, \mathbf{T}_{3}\right) \mathbf{T}_{1}^{2}\left(\mathbf{I}_{n}-\mathbf{T}_{2}^{2}\right)+p_{c_{2}, c_{3}}\left(\mathbf{T}_{2}, \mathbf{T}_{3}\right)\left(\mathbf{I}_{n}-\mathbf{T}_{3}^{2}\right),
\end{aligned}
$$

where $p_{a, b}: \mathbb{C}^{2} \rightarrow \mathbb{C}$ and $q: \mathbb{C}^{3} \rightarrow \mathbb{C}$ are the following complex polynomials,

$$
\begin{gathered}
p_{a, b}(z, w)=\frac{b^{2}}{a\left(a^{2}-b^{2}\right)} z w^{2}+\frac{a^{2}}{b\left(a^{2}-b^{2}\right)} z^{2} w+\frac{1}{a} z+\frac{1}{b} w, \quad\left(a, b \in \mathbb{C}, a^{2} \neq b^{2}\right), \\
q(z, w, u)=\frac{\left(c_{1}^{3}-c_{1} c_{2}^{2}-c_{1} c_{3}^{2}\right) z+\left(c_{2}^{3}-c_{2} c_{3}^{2}-c_{2} c_{1}^{2}\right) w+\left(c_{3}^{3}-c_{3} c_{1}^{2}-c_{3} c_{2}^{2}\right) u}{\left(c_{1}+c_{2}+c_{3}\right)\left(c_{1}+c_{2}-c_{3}\right)\left(c_{1}-c_{2}+c_{3}\right)\left(c_{1}-c_{2}-c_{3}\right)}
\end{gathered}
$$

In particular, if $\mathbf{T}_{1}^{2}+\mathbf{T}_{2}^{2}+\mathbf{T}_{3}^{2}$ is nonsingular, then $c_{1} \mathbf{T}_{1}+c_{2} \mathbf{T}_{2}+c_{3} \mathbf{T}_{3}$ is nonsingular and $\left(c_{1} \mathbf{T}_{1}+c_{2} \mathbf{T}_{2}+c_{3} \mathbf{T}_{3}\right)^{-1}$ is given by (2.6).

Proof. By [12, Exercise 5.10.12], there exist nonsingular matrices $\mathbf{S}_{1} \in \mathcal{M}_{n}$ and $\mathbf{X}_{1} \in \mathcal{M}_{n-t}$ such that $\mathbf{T}_{1}=\mathbf{S}_{1}\left(\mathbf{X}_{1} \oplus \mathbf{0}\right) \mathbf{S}_{1}^{-1}$. The tripotency of $\mathbf{T}_{1}$ and the nonsingularity of $\mathbf{X}_{1}$ leads to $\mathbf{X}_{1}^{2}=\mathbf{I}_{n-t}$. As $\mathbf{T}_{1} \mathbf{T}_{2}=\mathbf{T}_{2} \mathbf{T}_{1}$ and $\mathbf{T}_{1} \mathbf{T}_{3}=\mathbf{T}_{3} \mathbf{T}_{1}$, we can write matrices $\mathbf{T}_{2}$ and $\mathbf{T}_{3}$ as follows

$$
\mathbf{T}_{2}=\mathbf{S}_{1}\left(\begin{array}{cc}
\mathbf{X}_{2} & \mathbf{0} \\
\mathbf{0} & \mathbf{D}_{2}
\end{array}\right) \mathbf{S}_{1}^{-1}, \quad \mathbf{T}_{3}=\mathbf{S}_{1}\left(\begin{array}{cc}
\mathbf{X}_{3} & \mathbf{0} \\
\mathbf{0} & \mathbf{D}_{3}
\end{array}\right) \mathbf{S}_{1}^{-1}, \quad \mathbf{D}_{2}, \mathbf{D}_{3} \in \mathcal{M}_{t},
$$

83 with

$$
\mathbf{X}_{1} \mathbf{X}_{2}=\mathbf{X}_{2} \mathbf{X}_{1}, \quad \mathbf{X}_{1} \mathbf{X}_{3}=\mathbf{X}_{3} \mathbf{X}_{1}
$$

Let us notice that matrices $\mathbf{X}_{2}, \mathbf{X}_{3}, \mathbf{D}_{2}, \mathbf{D}_{3}$ are tripotent because $\mathbf{T}_{2}$ and $\mathbf{T}_{3}$ are tripotent. By applying again exercise [12, Exercise 5.10.12], there exist nonsingular matrices $\mathbf{S}_{2} \in$ $\mathcal{M}_{n-t}$ and $\mathbf{Y}_{2} \in \mathcal{M}_{n-t-s}$ such that $\mathbf{X}_{2}=\mathbf{S}_{2}\left(\mathbf{Y}_{2} \oplus \mathbf{0}\right) \mathbf{S}_{2}^{-1}$. From (2.7) we can write

$$
\mathbf{X}_{1}=\mathbf{S}_{2}\left(\begin{array}{cc}
\mathbf{Y}_{1} & \mathbf{0} \\
\mathbf{0} & \mathbf{C}_{1}
\end{array}\right) \mathbf{S}_{2}^{-1}, \quad \mathbf{X}_{3}=\mathbf{S}_{2}\left(\begin{array}{cc}
\mathbf{Y}_{3} & \mathbf{0} \\
\mathbf{0} & \mathbf{C}_{3}
\end{array}\right) \mathbf{S}_{2}^{-1}
$$

${ }_{84}$ Observe that $\mathbf{Y}_{1}^{2}=I_{n-t-s}, \mathbf{C}_{1}^{2}=I_{s}, \mathbf{Y}_{3}^{3}=\mathbf{Y}_{3}$, and $\mathbf{C}_{3}^{3}=\mathbf{C}_{3}$.

Finally, utilize again [12, Exercise 5.10.12] to matrix $\mathbf{Y}_{3}$ to obtain nonsingular matrices $\mathbf{S}_{3} \in \mathcal{M}_{n-t-s}$ and $\mathbf{A}_{3} \in \mathcal{M}_{n-t-s-r}$ such that $\mathbf{Y}_{3}=\mathbf{S}_{3}\left(\mathbf{A}_{3} \oplus \mathbf{0}\right) \mathbf{S}_{3}^{-1}$. By carrying out the same routine as before, we can write

$$
\mathbf{Y}_{1}=\mathbf{S}_{3}\left(\begin{array}{cc}
\mathbf{A}_{1} & \mathbf{0} \\
\mathbf{0} & \mathbf{B}_{1}
\end{array}\right) \mathbf{S}_{3}^{-1}, \quad \mathbf{Y}_{2}=\mathbf{S}_{3}\left(\begin{array}{cc}
\mathbf{A}_{2} & \mathbf{0} \\
\mathbf{0} & \mathbf{B}_{2}
\end{array}\right) \mathbf{S}_{3}^{-1} .
$$

Let us define $m=n-t-s-r$. By setting $\mathbf{S}=\mathbf{S}_{1}\left(\mathbf{S}_{2} \oplus \mathbf{I}_{t}\right)\left(\mathbf{S}_{3} \oplus \mathbf{I}_{s} \oplus \mathbf{I}_{t}\right)$, one easily has

$$
\begin{gathered}
\mathbf{T}_{1}=\mathbf{S}\left(\mathbf{A}_{1} \oplus \mathbf{B}_{1} \oplus \mathbf{C}_{1} \oplus \mathbf{0}\right) \mathbf{S}^{-1}, \quad \mathbf{T}_{2}=\mathbf{S}\left(\mathbf{A}_{2} \oplus \mathbf{B}_{2} \oplus \mathbf{0} \oplus \mathbf{D}_{2}\right) \mathbf{S}^{-1}, \\
\mathbf{T}_{3}=\mathbf{S}\left(\mathbf{A}_{3} \oplus \mathbf{0} \oplus \mathbf{C}_{3} \oplus \mathbf{D}_{3}\right) \mathbf{S}^{-1} .
\end{gathered}
$$

and the matrices $\mathbf{A}_{1}, \mathbf{A}_{2}, \mathbf{A}_{3}, \mathbf{B}_{1}, \mathbf{B}_{2}$, and $\mathbf{C}_{1}$ are nonsingular. Observe that the tripotency of $\mathbf{T}_{i}$ leads to the tripotency of these matrices $\mathbf{A}_{i}, \mathbf{B}_{i}, \mathbf{C}_{i}$, and $\mathbf{D}_{i}$. Furthermore, since $\mathbf{A}_{1}, \mathbf{A}_{2}, \mathbf{A}_{3}, \mathbf{B}_{1}, \mathbf{B}_{2}$, and $\mathbf{C}_{1}$ are nonsingular, then $\mathbf{A}_{i}^{2}=\mathbf{I}_{m}$ (for $i=1,2,3$ ), $\mathbf{B}_{i}^{2}=\mathbf{I}_{r}$ (for $i=1,2)$ and $\mathbf{C}_{1}^{2}=\mathbf{I}_{s}$. In addition, the families $\left\{\mathbf{A}_{i}\right\}_{i=1,2,3},\left\{\mathbf{B}_{i}\right\}_{i=1,2},\left\{\mathbf{C}_{i}\right\}_{i=1,3}$, and $\left\{\mathbf{D}_{i}\right\}_{i=2,3}$ are commutative.

Observe that

$$
\mathbf{T}_{1}^{2}+\mathbf{T}_{2}^{2}+\mathbf{T}_{3}^{2}=\mathbf{S}\left(3 \mathbf{I}_{m} \oplus\left(\mathbf{B}_{1}^{2}+\mathbf{B}_{2}^{2}\right) \oplus\left(\mathbf{C}_{1}^{2}+\mathbf{C}_{3}^{2}\right) \oplus\left(\mathbf{D}_{2}^{2}+\mathbf{D}_{3}^{2}\right)\right) \mathbf{S}^{-1}
$$


and

$$
\begin{aligned}
& c_{1} \mathbf{T}_{1}+c_{2} \mathbf{T}_{2}+c_{3} \mathbf{T}_{3} \\
& \quad=\mathbf{S}\left(\left(c_{1} \mathbf{A}_{1}+c_{2} \mathbf{A}_{2}+c_{3} \mathbf{A}_{3}\right) \oplus\left(c_{1} \mathbf{B}_{1}+c_{2} \mathbf{B}_{2}\right) \oplus\left(c_{1} \mathbf{C}_{1}+c_{3} \mathbf{C}_{3}\right) \oplus\left(c_{2} \mathbf{D}_{2}+c_{3} \mathbf{D}_{3}\right)\right) \mathbf{S}^{-1} .
\end{aligned}
$$

By the equality given in (2.5) we have that $c_{1} \mathbf{A}_{1}+c_{2} \mathbf{A}_{2}+c_{3} \mathbf{A}_{3}$ is nonsingular and

$$
\left(c_{1} \mathbf{A}_{1}+c_{2} \mathbf{A}_{2}+c_{3} \mathbf{A}_{3}\right)^{-1}=q\left(\mathbf{A}_{1}, \mathbf{A}_{2}, \mathbf{A}_{3}\right) .
$$

Since $c_{1} \mathbf{A}_{1}+c_{2} \mathbf{A}_{2}+c_{3} \mathbf{A}_{3}$ is nonsingular, then $\mathcal{N}\left(c_{1} \mathbf{A}_{1}+c_{2} \mathbf{A}_{2}+c_{3} \mathbf{A}_{3}\right)=\mathcal{N}\left(3 \mathbf{I}_{m}\right)$ and $\mathcal{R}\left(c_{1} \mathbf{A}_{1}+c_{2} \mathbf{A}_{2}+c_{3} \mathbf{A}_{3}\right)=\mathcal{R}\left(3 \mathbf{I}_{m}\right)$. Theorem 2.1 leads to $\mathcal{N}\left(c_{1} \mathbf{B}_{1}+c_{2} \mathbf{B}_{2}\right)=\mathcal{N}\left(\mathbf{B}_{1}^{2}+\mathbf{B}_{2}^{2}\right)$, $\mathcal{N}\left(c_{1} \mathbf{C}_{1}+c_{3} \mathbf{C}_{3}\right)=\mathcal{N}\left(\mathbf{C}_{1}^{2}+\mathbf{C}_{3}^{2}\right), \mathcal{N}\left(c_{2} \mathbf{D}_{2}+c_{3} \mathbf{D}_{3}\right)=\mathcal{N}\left(\mathbf{D}_{2}^{2}+\mathbf{D}_{3}^{2}\right)$, and analogous identities for the range space. By considering (2.8), (2.9), and the first part of Lemma 2.2 we get that the null space (range space) of $c_{1} \mathbf{T}_{1}+c_{2} \mathbf{T}_{2}+c_{3} \mathbf{T}_{3}$ equals to the null space (range space) $\mathbf{T}_{1}^{2}+\mathbf{T}_{2}^{2}+\mathbf{T}_{3}^{2}$.

By Theorem 2.1 we have the group invertibility of $c_{1} \mathbf{B}_{1}+c_{2} \mathbf{B}_{2}, c_{1} \mathbf{C}_{1}+c_{3} \mathbf{C}_{3}$, and $c_{2} \mathbf{D}_{2}+c_{3} \mathbf{D}_{3}$. Also we get

$$
\left(c_{1} \mathbf{B}_{1}+c_{2} \mathbf{B}_{2}\right)^{\#}=p_{c_{1}, c_{2}}\left(\mathbf{B}_{1}, \mathbf{B}_{2}\right), \quad\left(c_{1} \mathbf{C}_{1}+c_{3} \mathbf{C}_{3}\right)^{\#}=p_{c_{1}, c_{3}}\left(\mathbf{C}_{1}, \mathbf{C}_{3}\right),
$$

and

$$
\left(c_{2} \mathbf{D}_{2}+c_{3} \mathbf{D}_{3}\right)^{\#}=p_{c_{2}, c_{3}}\left(\mathbf{D}_{2}, \mathbf{D}_{3}\right) \text {. }
$$

98 The second part of Lemma 2.2 leads to the group invertibility of $c_{1} \mathbf{T}_{1}+c_{2} \mathbf{T}_{2}+c_{3} \mathbf{T}_{3}$ and

$$
\begin{aligned}
\left(c_{1} \mathbf{T}_{1}\right. & \left.+c_{2} \mathbf{T}_{2}+c_{3} \mathbf{T}_{3}\right)^{\#} \\
& =\mathbf{S}\left[q\left(\mathbf{A}_{1}, \mathbf{A}_{2}, \mathbf{A}_{3}\right) \oplus p_{c_{1}, c_{2}}\left(\mathbf{B}_{1}, \mathbf{B}_{2}\right) \oplus p_{c_{1}, c_{3}}\left(\mathbf{C}_{1}, \mathbf{C}_{3}\right) \oplus p_{c_{2}, c_{3}}\left(\mathbf{D}_{2}, \mathbf{D}_{3}\right)\right] \mathbf{S}^{-1} .
\end{aligned}
$$

Now, observe that

$$
\begin{aligned}
\mathbf{S}\left[q\left(\mathbf{A}_{1}, \mathbf{A}_{2}, \mathbf{A}_{3}\right) \oplus \mathbf{0} \oplus \mathbf{0} \oplus \mathbf{0}\right] \mathbf{S}^{-1} & =q\left(\mathbf{T}_{1}, \mathbf{T}_{2}, \mathbf{T}_{3}\right) \mathbf{S}\left(\mathbf{I}_{m} \oplus \mathbf{0} \oplus \mathbf{0} \oplus \mathbf{0}\right) \mathbf{S}^{-1} \\
& =q\left(\mathbf{T}_{1}, \mathbf{T}_{2}, \mathbf{T}_{3}\right) \mathbf{T}_{1}^{2} \mathbf{T}_{2}^{2} \mathbf{T}_{3}^{3} .
\end{aligned}
$$

Since $\mathbf{S}\left(\mathbf{0} \oplus \mathbf{I}_{r} \oplus \mathbf{0} \oplus \mathbf{0}\right) \mathbf{S}^{-1}=\mathbf{T}_{1}^{2} \mathbf{T}_{2}^{2}-\mathbf{T}_{1}^{2} \mathbf{T}_{2}^{2} \mathbf{T}_{3}^{2}=\mathbf{T}_{1}^{2} \mathbf{T}_{2}^{2}\left(\mathbf{I}_{n}-\mathbf{T}_{3}^{2}\right)$, we have

$$
\mathbf{S}\left[\mathbf{0} \oplus p_{c_{1}, c_{2}}\left(\mathbf{B}_{1}, \mathbf{B}_{2}\right) \oplus \mathbf{0} \oplus \mathbf{0}\right] \mathbf{S}^{-1}=p_{c_{1}, c_{2}}\left(\mathbf{T}_{1}, \mathbf{T}_{2}\right) \mathbf{T}_{1}^{2} \mathbf{T}_{2}^{2}\left(\mathbf{I}_{n}-\mathbf{T}_{3}^{2}\right) .
$$

Another two useful idempotents are the following two matrices: $\mathbf{S}\left(\mathbf{0} \oplus \mathbf{0} \oplus \mathbf{I}_{s} \oplus \mathbf{0}\right) \mathbf{S}^{-1}=$ $\mathbf{T}_{1}^{2}-\mathbf{T}_{1}^{2} \mathbf{T}_{1}^{2}=\mathbf{T}_{1}^{2}\left(\mathbf{I}_{n}-\mathbf{T}_{2}^{2}\right)$ and $\mathbf{S}\left(\mathbf{0} \oplus \mathbf{0} \oplus \mathbf{0} \oplus \mathbf{I}_{t}\right) \mathbf{S}^{-1}=\mathbf{I}_{n}-\mathbf{T}_{1}^{2}$. Thus we have

$$
\mathbf{S}\left[\mathbf{0} \oplus \mathbf{0} \oplus p_{c_{1}, c_{3}}\left(\mathbf{C}_{1}, \mathbf{C}_{3}\right) \oplus \mathbf{0}\right] \mathbf{S}^{-1}=p_{c_{1}, c_{3}}\left(\mathbf{T}_{1}, \mathbf{T}_{3}\right) \mathbf{T}_{1}^{2}\left(\mathbf{I}_{n}-\mathbf{T}_{2}^{2}\right)
$$

and

$$
\mathbf{S}\left[\mathbf{0} \oplus \mathbf{0} \oplus \mathbf{0} \oplus p_{c_{2}, c_{3}}\left(\mathbf{D}_{2}, \mathbf{D}_{3}\right)\right] \mathbf{S}^{-1}=p_{c_{2}, c_{3}}\left(\mathbf{T}_{2}, \mathbf{T}_{3}\right)\left(\mathbf{I}_{n}-\mathbf{T}_{1}^{2}\right) .
$$

Considering (2.10)-(2.14) finishes the proof.

As we already pointed out, in this paper, similar results to the ones obtained in [10] are established for three tripotent or group invertible matrices.

Theorem 2.3. Let $\mathbf{T}_{1}, \mathbf{T}_{2}$, and $\mathbf{T}_{3} \in \mathcal{M}_{n}$ be three mutually commuting tripotent matrices. Then $\mathbf{T}_{1}+\mathbf{T}_{2}+\mathbf{T}_{3}$ is nonsingular if and only if $\mathbf{I}_{n}+\mathbf{T}_{1} \mathbf{T}_{2}+\mathbf{T}_{2} \mathbf{T}_{3}+\mathbf{T}_{3} \mathbf{T}_{1}+\mathbf{T}_{1} \mathbf{T}_{2} \mathbf{T}_{3}$ and $\mathbf{T}_{1}^{2}+\mathbf{T}_{2}^{2}+\mathbf{T}_{3}^{2}$ are nonsingular.

Proof. Since $\mathbf{T}_{1}, \mathbf{T}_{2}$, and $\mathbf{T}_{3}$ are tripotent and mutually commutating, they are simultaneously diagonalizable (see, e.g., [7, page 52]). Hence there is a single similarity matrix $\mathbf{S} \in \mathcal{M}_{n}$ such that $\mathbf{T}_{1}=\mathbf{S} \operatorname{diag}\left(\lambda_{1}, \lambda_{2}, \ldots, \lambda_{n}\right) \mathbf{S}^{-1}, \mathbf{T}_{2}=\mathbf{S} \operatorname{diag}\left(\mu_{1}, \mu_{2}, \ldots, \mu_{n}\right) \mathbf{S}^{-1}$ and 
$\mathbf{T}_{3}=\mathbf{S} \operatorname{diag}\left(\gamma_{1}, \gamma_{2}, \ldots, \gamma_{n}\right) \mathbf{S}^{-1}$ being $\left\{\lambda_{i}\right\}_{i=1}^{n},\left\{\mu_{i}\right\}_{i=1}^{n}$ and $\left\{\gamma_{i}\right\}_{i=1}^{n}$ the sets of eigenvalues of $\mathbf{T}_{1}, \mathbf{T}_{2}$ and $\mathbf{T}_{3}$, with proper multiplicities, respectively. On the other hand,

$$
\mathbf{T}_{1}+\mathbf{T}_{2}+\mathbf{T}_{3}=\mathbf{S} \operatorname{diag}\left(\lambda_{1}+\mu_{1}+\gamma_{1}, \ldots, \lambda_{n}+\mu_{n}+\gamma_{n}\right) \mathbf{S}^{-1},
$$

$$
\mathbf{I}_{n}+\mathbf{T}_{1} \mathbf{T}_{2}+\mathbf{T}_{2} \mathbf{T}_{3}+\mathbf{T}_{3} \mathbf{T}_{1}+\mathbf{T}_{1} \mathbf{T}_{2} \mathbf{T}_{3}=\mathbf{S} \operatorname{diag}\left(p\left(\lambda_{1}, \mu_{1}, \gamma_{1}\right), \ldots, p\left(\lambda_{n}, \mu_{n}, \gamma_{n}\right)\right) \mathbf{S}^{-1}
$$

and

$$
\mathbf{T}_{1}^{2}+\mathbf{T}_{2}^{2}+\mathbf{T}_{3}^{2}=\mathbf{S} \operatorname{diag}\left(\lambda_{1}^{2}+\mu_{1}^{2}+\gamma_{1}^{2}, \ldots, \lambda_{n}^{2}+\mu_{n}^{2}+\gamma_{n}^{2}\right) \mathbf{S}^{-1},
$$

where $p: \mathbb{C}^{3} \rightarrow \mathbb{C}$ is given by $p(z, w, u)=1+z w+w u+u z+z w u$.

Assume that $\mathbf{T}_{1}+\mathbf{T}_{2}+\mathbf{T}_{3}$ is nonsingular. From (2.15), we get $\lambda_{i}+\mu_{i}+\gamma_{i} \neq 0$ for any $i=1, \ldots, n$ and hence

$$
\left(\lambda_{i}, \mu_{i}, \gamma_{i}\right) \in \Phi^{3} \backslash\{(-1,1,0),(0,-1,1),(-1,0,1),(0,0,0),(1,0,-1),(0,1,-1),(1,-1,0)\}
$$

for all $i=1,2, \ldots, n$, where $\Phi=\{-1,0,1\}$. Therefore, it is obtained that $p\left(\lambda_{i}, \mu_{i}, \gamma_{i}\right) \neq 0$ and $\lambda_{i}^{2}+\mu_{i}^{2}+\gamma_{i}^{2} \neq 0$ for all $i=1,2, \ldots, n$. In view of (2.16) and (2.17) it is seen that $\mathbf{I}_{n}+\mathbf{T}_{1} \mathbf{T}_{2}+\mathbf{T}_{2} \mathbf{T}_{3}+\mathbf{T}_{3} \mathbf{T}_{1}+\mathbf{T}_{1} \mathbf{T}_{2} \mathbf{T}_{3}$ and $\mathbf{T}_{1}^{2}+\mathbf{T}_{2}^{2}+\mathbf{T}_{3}^{2}$ are nonsingular.

Now, assume that $\mathbf{I}_{n}+\mathbf{T}_{1} \mathbf{T}_{2}+\mathbf{T}_{2} \mathbf{T}_{3}+\mathbf{T}_{3} \mathbf{T}_{1}+\mathbf{T}_{1} \mathbf{T}_{2} \mathbf{T}_{3}$ and $\mathbf{T}_{1}^{2}+\mathbf{T}_{2}^{2}+\mathbf{T}_{3}^{2}$ are nonsingular. From the nonsingularity of the first matrix we get

$$
1+\lambda_{i} \mu_{i}+\mu_{i} \gamma_{i}+\gamma_{i} \lambda_{i}+\lambda_{i} \mu_{i} \gamma_{i} \neq 0 \quad \text { for all } i=1,2, \ldots, n \text {. }
$$

If $\mathbf{T}_{1}+\mathbf{T}_{2}+\mathbf{T}_{3}$ were singular, then there would exist some $j \in\{1,2, \ldots, n\}$ such that $\lambda_{j}+\mu_{j}+\gamma_{j}=0$. So, the unique solution satisfying simultaneously these two equations would be $\left(\lambda_{j}, \mu_{j}, \gamma_{j}\right)=(0,0,0)$. Hence, $\lambda_{j}^{2}+\mu_{j}^{2}+\gamma_{j}^{2}=0$ which would contradict to the assumption of the nonsingularity of $\mathbf{T}_{1}^{2}+\mathbf{T}_{2}^{2}+\mathbf{T}_{3}^{2}$. So the proof is complete.

Remark 2.2. It is evident that for a given $\mathbf{X} \in \mathcal{M}_{n}$, then $\mathbf{X}$ is tripotent if and only if $-\mathbf{X}$ is tripotent. Thus, by means of Theorem 2.3 , we can characterize the nonsingularity of $\varepsilon_{1} \mathbf{T}_{1}+\varepsilon_{2} \mathbf{T}_{2}+\varepsilon_{3} \mathbf{T}_{3}$, where $\varepsilon_{1}, \varepsilon_{1}, \varepsilon_{1} \in\{-1,1\}$ and $\mathbf{T}_{1}, \mathbf{T}_{2}, \mathbf{T}_{3} \in \mathcal{M}_{n}$ are tripotent matrices.

Remark 2.3. Let $p: \mathbb{C}^{3} \longrightarrow \mathbb{C}$ be the following complex polynomial:

$$
p(z, w, t)=\sum_{\substack{i, j, k=0 \\(i, j, k) \neq(0,0,0)}}^{m} c_{i, j, k} z^{i} w^{j} t^{k},
$$

where $m \in \mathbb{Z}^{+}, c_{i, j, k} \in \mathbb{C}$. Let $\mathbf{T}_{1}, \mathbf{T}_{2}$, and $\mathbf{T}_{3} \in \mathcal{M}_{n}$ be three mutually commuting tripotent matrices. Then,

$$
p\left(\mathbf{T}_{1}, \mathbf{T}_{2}, \mathbf{T}_{3}\right)=\mathbf{S} \operatorname{diag}\left[p\left(\lambda_{1}, \mu_{1}, \gamma_{1}\right), \ldots, p\left(\lambda_{n}, \mu_{n}, \gamma_{n}\right)\right] \mathbf{S}^{-1} .
$$

If $\mathbf{T}_{1}^{2}+\mathbf{T}_{2}^{2}+\mathbf{T}_{3}^{2}$ were singular, then there would exist $j \in\{1, \ldots, n\}$ satisfying $\lambda_{j}^{2}+\mu_{j}^{2}+\gamma_{j}^{2}=0$. Therefore, $\lambda_{j}=\mu_{j}=\gamma_{j}=0$. So, $p\left(\mathbf{T}_{1}, \mathbf{T}_{2}, \mathbf{T}_{3}\right)$ is singular because $p(0,0,0)=0$.

Hence, the following corollary can be given.

Corollary 2.2. Let $\mathbf{T}_{1}, \mathbf{T}_{2}$, and $\mathbf{T}_{3} \in \mathcal{M}_{n}$ be three mutually commuting tripotent matrices. If $\mathbf{I}_{n}+\mathbf{T}_{1} \mathbf{T}_{2}+\mathbf{T}_{2} \mathbf{T}_{3}+\mathbf{T}_{3} \mathbf{T}_{1}+\mathbf{T}_{1} \mathbf{T}_{2} \mathbf{T}_{3}$ is nonsingular and there exists a polynomial $p$ as in (2.18) such that $p\left(\mathbf{T}_{1}, \mathbf{T}_{2}, \mathbf{T}_{3}\right)$ is nonsingular, then $\mathbf{T}_{1}+\mathbf{T}_{2}+\mathbf{T}_{3}$ is nonsingular.

The next theorem is presented under weaker assumptions than the previous theorem.

Theorem 2.4. Let $\mathbf{T}_{1}, \mathbf{T}_{2}$, and $\mathbf{T}_{3} \in \mathcal{M}_{n}$ such that $\mathbf{T}_{1}$ is group invertible and $\mathbf{I}_{n}-\mathbf{T}_{1}^{\#} \mathbf{T}_{2}-$ $\mathbf{T}_{1}^{\#} \mathbf{T}_{3}$ is nonsingular. If one of the below conditions holds,

(i) if $\mathbf{T}_{2} \mathbf{T}_{1} \mathbf{T}_{1}^{\#}=\mathbf{T}_{2}, \mathbf{T}_{3} \mathbf{T}_{1} \mathbf{T}_{1}^{\#}=\mathbf{T}_{3}$, and there exists a polynomial $p$ in three variables not necessarily commutatative such that $p(0,0,0)=0$ and $p\left(\mathbf{T}_{1}, \mathbf{T}_{2}, \mathbf{T}_{3}\right)$ is nonsingular, 
(ii) if $\mathbf{T}_{2} \mathbf{T}_{1} \mathbf{T}_{1}^{\#}=\mathbf{T}_{1} \mathbf{T}_{1}^{\#} \mathbf{T}_{2}, \mathbf{T}_{3} \mathbf{T}_{1} \mathbf{T}_{1}^{\#}=\mathbf{T}_{3}$, and there exists a polynomial $p$ in three variables not necessarily commutatative such that $p(0,0,0)=0$ and $p\left(\mathbf{T}_{1}, \mathbf{T}_{1} \mathbf{T}_{2}, \mathbf{T}_{3}\right)$ is nonsingular,

(iii) if $\mathbf{T}_{2} \mathbf{T}_{1} \mathbf{T}_{1}^{\#}=\mathbf{T}_{1} \mathbf{T}_{1}^{\#} \mathbf{T}_{2}, \mathbf{T}_{3} \mathbf{T}_{1} \mathbf{T}_{1}^{\#}=\mathbf{T}_{1} \mathbf{T}_{1}^{\#} \mathbf{T}_{3}$, and there exists a polynomial $p$ in three variables not necessarily commutatative such that $p(0,0,0)=0$ and $p\left(\mathbf{T}_{1}, \mathbf{T}_{1} \mathbf{T}_{2}, \mathbf{T}_{1} \mathbf{T}_{3}\right)$ is nonsingular,

then $\mathbf{T}_{1}-\mathbf{T}_{2}-\mathbf{T}_{3}$ is nonsingular.

Proof. Let $\mathbf{x} \in \mathcal{N}\left(\mathbf{T}_{1}-\mathbf{T}_{2}-\mathbf{T}_{3}\right)$, i.e.,

$$
\mathbf{T}_{1} \mathbf{x}=\left(\mathbf{T}_{2}+\mathbf{T}_{3}\right) \mathbf{x}
$$

(i) Assume that the conditions given in (i) are satisfied. Premultiplying (2.19) by $\mathbf{T}_{1} \mathbf{T}_{1}^{\#}$, $\mathbf{T}_{2} \mathbf{T}_{1}^{\#}, \mathbf{T}_{3} \mathbf{T}_{1}^{\#}$, it is obtained $\mathbf{T}_{1} \mathbf{x}=\mathbf{T}_{1} \mathbf{T}_{1}^{\#}\left(\mathbf{T}_{2}+\mathbf{T}_{3}\right) \mathbf{x}, \mathbf{T}_{2} \mathbf{x}=\mathbf{T}_{2} \mathbf{T}_{1}^{\#}\left(\mathbf{T}_{2}+\mathbf{T}_{3}\right) \mathbf{x}$, and $\mathbf{T}_{3} \mathbf{x}=\mathbf{T}_{3} \mathbf{T}_{1}^{\#}\left(\mathbf{T}_{2}+\mathbf{T}_{3}\right) \mathbf{x}$, respectively. If these equations are reorganized, we get

$$
\mathbf{T}_{1}\left(\mathbf{I}_{n}-\mathbf{T}_{1}^{\#}\left(\mathbf{T}_{2}+\mathbf{T}_{3}\right) \mathbf{x}=\mathbf{T}_{2}\left(\mathbf{I}_{n}-\mathbf{T}_{1}^{\#}\left(\mathbf{T}_{2}+\mathbf{T}_{3}\right) \mathbf{x}=\mathbf{T}_{2}\left(\mathbf{I}_{n}-\mathbf{T}_{1}^{\#}\left(\mathbf{T}_{2}+\mathbf{T}_{3}\right) \mathbf{x}=\mathbf{0} .\right.\right.\right.
$$

There exists three polynomials in three variables not necessarily commutative, say $p_{1}, p_{2}$, and $p_{3}$, such that $p\left(\mathbf{T}_{1}, \mathbf{T}_{2}, \mathbf{T}_{3}\right)=p_{1}\left(\mathbf{T}_{1}, \mathbf{T}_{2}, \mathbf{T}_{3}\right) \mathbf{T}_{1}+p_{2}\left(\mathbf{T}_{1}, \mathbf{T}_{2}, \mathbf{T}_{3}\right) \mathbf{T}_{2}+p_{3}\left(\mathbf{T}_{1}, \mathbf{T}_{2}, \mathbf{T}_{3}\right) \mathbf{T}_{3}$. Thus from (2.20) it is obtained

$$
\begin{aligned}
& p\left(\mathbf{T}_{1}, \mathbf{T}_{2}, \mathbf{T}_{3}\right)\left[\mathbf{I}_{n}-\mathbf{T}_{1}^{\#}\left(\mathbf{T}_{2}+\mathbf{T}_{3}\right] \mathbf{x}\right. \\
& \quad=\left[p_{1}\left(\mathbf{T}_{1}, \mathbf{T}_{2}, \mathbf{T}_{3}\right) \mathbf{T}_{1}+p_{2}\left(\mathbf{T}_{1}, \mathbf{T}_{2}, \mathbf{T}_{3}\right) \mathbf{T}_{2}+p_{3}\left(\mathbf{T}_{1}, \mathbf{T}_{2}, \mathbf{T}_{3}\right) \mathbf{T}_{3}\right]\left[\mathbf{I}_{n}-\mathbf{T}_{1}^{\#}\left(\mathbf{T}_{2}+\mathbf{T}_{3}\right)\right] \mathbf{x} \\
& \quad=\mathbf{0}
\end{aligned}
$$

Under the assumption that $\mathbf{I}_{n}-\mathbf{T}_{1}^{\#} \mathbf{T}_{2}-\mathbf{T}_{1}^{\#} \mathbf{T}_{3}$ and $p\left(\mathbf{T}_{1}, \mathbf{T}_{2}, \mathbf{T}_{3}\right)$ are nonsingular, the above computation yields $\mathbf{x}=\mathbf{0}$, which means that $\mathbf{T}_{1}-\mathbf{T}_{2}-\mathbf{T}_{3}$ is nonsingular. So the proof of item (i) is complete.

(ii) By premultiplying (2.19) by $\mathbf{T}_{1} \mathbf{T}_{1}^{\#}, \mathbf{T}_{1} \mathbf{T}_{2} \mathbf{T}_{1}^{\#}$, and $\mathbf{T}_{3} \mathbf{T}_{1}^{\#}$ it follows that $\mathbf{T}_{1} \mathbf{x}=$ $\mathbf{T}_{1} \mathbf{T}_{1}^{\#}\left(\mathbf{T}_{2}+\mathbf{T}_{3}\right) \mathbf{x}, \mathbf{T}_{1} \mathbf{T}_{2} \mathbf{x}=\mathbf{T}_{1} \mathbf{T}_{2} \mathbf{T}_{1}^{\#}\left(\mathbf{T}_{2}+\mathbf{T}_{3}\right) \mathbf{x}$, and $\mathbf{T}_{3} \mathbf{x}=\mathbf{T}_{3} \mathbf{T}_{1}^{\#}\left(\mathbf{T}_{2}+\mathbf{T}_{3}\right) \mathbf{x}$, respectively. From these identities we obtain

$\mathbf{T}_{1}\left(\mathbf{I}_{n}-\mathbf{T}_{1}^{\#}\left(\mathbf{T}_{2}+\mathbf{T}_{3}\right) \mathbf{x}=\mathbf{T}_{1} \mathbf{T}_{2}\left(\mathbf{I}_{n}-\mathbf{T}_{1}^{\#}\left(\mathbf{T}_{2}+\mathbf{T}_{3}\right) \mathbf{x}=\mathbf{T}_{3}\left(\mathbf{I}_{n}-\mathbf{T}_{1}^{\#}\left(\mathbf{T}_{2}+\mathbf{T}_{3}\right) \mathbf{x}=\mathbf{0}\right.\right.\right.$.

Since $p(0,0,0)=0$, there exist three polynomials $p_{1}, p_{2}, p_{3}$ in three noncommuting variables such that

$$
p\left(z_{1}, z_{2}, z_{3}\right)=p_{1}\left(z_{1}, z_{2}, z_{3}\right) z_{1}+p_{2}\left(z_{1}, z_{1} z_{2}, z_{3}\right) z_{1} z_{2}+p_{3}\left(z_{1}, z_{2}, z_{3}\right) z_{3},
$$

By carrying out as in the proof of item (i), we can prove (ii).

Item (iii) can be proved in a similar way as in the proofs of items (i) and (ii).

Remark 2.4. Let $\mathbf{T}_{1} \in \mathcal{M}_{n}$ be group invertible and $\mathbf{A} \in \mathcal{M}_{n}$. The conditions $\mathbf{A T}_{1} \mathbf{T}_{1}^{\#}=\mathbf{A}$ and $\mathbf{A} \mathbf{T}_{1} \mathbf{T}_{1}^{\#}=\mathbf{T}_{1} \mathbf{T}_{1}^{\#} \mathbf{A}$ appearing in Theorem 2.4 are independent. In fact, we can write $\mathbf{T}_{1}=\mathbf{S}(\mathbf{K} \oplus \mathbf{0}) \mathbf{S}^{-1}$ for some nonsingular matrices $\mathbf{S} \in \mathcal{M}_{n}, \mathbf{K} \in \mathcal{M}_{r}$, being $r=\operatorname{rank}\left(\mathbf{T}_{1}\right)$. By writing

$$
\mathbf{A}=\mathbf{S}\left(\begin{array}{cc}
\mathbf{X} & \mathbf{Y} \\
\mathbf{Z} & \mathbf{T}
\end{array}\right) \mathbf{S}^{-1}, \quad \mathbf{X} \in \mathcal{M}_{r}
$$

and using the nonsingularity of $\mathbf{K}$, one has

$$
\mathbf{A T}_{1} \mathbf{T}_{1}^{\#}=\mathbf{T}_{1} \mathbf{T}_{1}^{\#} \mathbf{A} \quad \Longleftrightarrow \quad \mathbf{Y}=\mathbf{0} \text { and } \mathbf{Z}=\mathbf{0},
$$


and

$$
\mathbf{A T}_{1} \mathbf{T}_{1}^{\#}=\mathbf{A} \quad \Longleftrightarrow \quad \mathbf{Y}=\mathbf{0} \text { and } \mathbf{T}=\mathbf{0} .
$$

The first of the two above conditions is related to the so-called sharp ordering, introduced by Mitra [13] in 1987 (for a recent survey of matrix orderings, see [14]) is defined in the subset of $\mathcal{M}_{n}$ composed of group invertible matrices by

$$
\mathbf{M} \stackrel{\#}{\leq} \mathbf{N} \quad \Longleftrightarrow \quad \mathbf{M}^{\#} \mathbf{M}=\mathbf{M}^{\#} \mathbf{N} \text { and } \mathbf{M M}^{\#}=\mathbf{N M}^{\#} .
$$

As is easy to see, if $\mathbf{T}_{1}$ is written as $\mathbf{T}_{1}=\mathbf{S}(\mathbf{K} \oplus \mathbf{0}) \mathbf{S}^{-1}$ and $\mathbf{A}$ is written as in (2.21), then

$$
\mathbf{T}_{1} \stackrel{\#}{\leq} \mathbf{A} \quad \Longleftrightarrow \mathbf{X}=\mathbf{K}, \mathbf{Y}=\mathbf{0} \text {, and } \mathbf{Z}=\mathbf{0},
$$

164

then $\left(c_{1} \mathbf{T}_{1}+c_{2} \mathbf{T}_{2}+c_{3} \mathbf{T}_{3}\right)^{-1}$ is nonsingular and

$$
\begin{aligned}
& \left(c_{1} \mathbf{T}_{1}+c_{2} \mathbf{T}_{2}+c_{3} \mathbf{T}_{3}\right)^{-1} \\
& \quad=\left[\left(c_{1}^{-1}-\delta\right) \mathbf{T}_{1} \mathbf{T}_{1}^{\#}+\left(c_{2}^{-1}-\delta\right) \mathbf{T}_{2} \mathbf{T}_{2}^{\#}+\left(c_{3}^{-1}-\delta\right) \mathbf{T}_{3} \mathbf{T}_{3}^{\#}+\delta \mathbf{I}_{n}\right]\left(\mathbf{T}_{1}+\mathbf{T}_{2}+\mathbf{T}_{3}\right)^{-1}
\end{aligned}
$$

Proof. Let $\alpha=c_{1}^{-1}-\delta, \beta=c_{2}^{-1}-\delta$, and $\gamma=c_{3}^{-1}-\delta$. The proof of this theorem is immediately seen from the following equality:

$$
\left(c_{1} \mathbf{T}_{1}+c_{2} \mathbf{T}_{2}+c_{3} \mathbf{T}_{3}\right)\left(\alpha \mathbf{T}_{1} \mathbf{T}_{1}^{\#}+\beta \mathbf{T}_{2} \mathbf{T}_{2}^{\#}+\gamma \mathbf{T}_{3} \mathbf{T}_{3}^{\#}+\delta \mathbf{I}_{n}\right)=\mathbf{T}_{1}+\mathbf{T}_{2}+\mathbf{T}_{3} .
$$

It can be given some kind of the converse of Theorem 2.4 in case that $\mathbf{T}_{1}, \mathbf{T}_{2}, \mathbf{T}_{3} \in \mathcal{M}_{n}$ are three mutually commuting group invertible matrices satisfying $\mathbf{T}_{1} \mathbf{T}_{2} \mathbf{T}_{3}=\mathbf{T}_{1}^{2} \mathbf{T}_{2}-$ $\mathbf{T}_{2}^{2} \mathbf{T}_{1}=\mathbf{T}_{2}^{2} \mathbf{T}_{3}+\mathbf{T}_{3}^{2} \mathbf{T}_{2}=\mathbf{T}_{1}^{2} \mathbf{T}_{3}-\mathbf{T}_{3}^{2} \mathbf{T}_{1}$. Then

$$
\left(\mathbf{T}_{1}-\mathbf{T}_{2}-\mathbf{T}_{3}\right) \mathbf{T}_{1} \mathbf{T}_{2}=\left(\mathbf{T}_{1}-\mathbf{T}_{2}-\mathbf{T}_{3}\right) \mathbf{T}_{3} \mathbf{T}_{1}=\left(\mathbf{T}_{1}-\mathbf{T}_{2}-\mathbf{T}_{3}\right) \mathbf{T}_{2} \mathbf{T}_{3}=\mathbf{0},
$$

and hence the invertibility of $\mathbf{T}_{1}-\mathbf{T}_{2}-\mathbf{T}_{3}$ leads to $\mathbf{T}_{1} \mathbf{T}_{2}=\mathbf{T}_{3} \mathbf{T}_{1}=\mathbf{T}_{2} \mathbf{T}_{3}=\mathbf{0}$. Thus it can be written $c_{1} \mathbf{T}_{1}+c_{2} \mathbf{T}_{2}+c_{3} \mathbf{T}_{3}-c_{4}\left(\mathbf{T}_{1} \mathbf{T}_{2}+\mathbf{T}_{3} \mathbf{T}_{1}+\mathbf{T}_{2} \mathbf{T}_{3}\right)=c_{1} \mathbf{T}_{1}+c_{2} \mathbf{T}_{2}+c_{3} \mathbf{T}_{3}$, and it be given the explicit expression of $\left(c_{1} \mathbf{T}_{1}+c_{2} \mathbf{T}_{2}+c_{3} \mathbf{T}_{3}\right)^{-1}$ in terms of $\left(\mathbf{T}_{1}-\mathbf{T}_{2}-\mathbf{T}_{3}\right)^{-1}$

Theorem 2.5. Let $c_{1}, c_{2}, c_{3} \in \mathbb{C}^{*}$ and $\mathbf{T}_{1}, \mathbf{T}_{2}$, and $\mathbf{T}_{3} \in \mathcal{M}_{n}$ be three group invertible matrices such that $\mathbf{T}_{1}+\mathbf{T}_{2}+\mathbf{T}_{3}$ is nonsingular. If there exists $\delta \in \mathbb{C}$ such that

$$
c_{1}\left(c_{2}^{-1}-\delta\right) \mathbf{T}_{1} \mathbf{T}_{2} \mathbf{T}_{2}^{\#}+c_{2}\left(c_{1}^{-1}-\delta\right) \mathbf{T}_{2} \mathbf{T}_{1} \mathbf{T}_{1}^{\#}=\mathbf{0},
$$

and

$$
c_{2}\left(c_{3}^{-1}-\delta\right) \mathbf{T}_{2} \mathbf{T}_{3} \mathbf{T}_{3}^{\#}+c_{3}\left(c_{2}^{-1}-\delta\right) \mathbf{T}_{3} \mathbf{T}_{2} \mathbf{T}_{2}^{\#}=\mathbf{0},
$$

The above Theorem 2.5 permits establish many corollaries. As an exemplary list we can state two some of them in the foregoing paragraphs:

Let $c_{1}, c_{2} \in \mathbb{C}^{*}$ and $\mathbf{T}_{1}, \mathbf{T}_{2} \in \mathcal{M}_{n}$ be two group invertible matrices such that $\mathbf{T}_{1}+\mathbf{T}_{2}$ is nonsingular and $\mathbf{T}_{1} \mathbf{T}_{2} \mathbf{T}_{2}^{\#}=\lambda \mathbf{T}_{2} \mathbf{T}_{1} \mathbf{T}_{1}^{\#}$ for some $\lambda \in \mathbb{C}$. By setting $\mathbf{T}_{3}=\mathbf{0}$, obviously (2.23) and (2.24) hold. If exists $\delta \in \mathbb{C}$ such that (2.22) holds then

$$
\left|\begin{array}{cc}
c_{1}\left(c_{2}^{-1}-\delta\right) & c_{2}\left(c_{1}^{-1}-\delta\right) \\
-1 & \lambda
\end{array}\right|=0 .
$$


By expanding (2.25), one has $\lambda c_{1} c_{2}^{-1}-c_{2} c_{1}^{-1}=\left(\lambda c_{1}-c_{2}\right) \delta$. Thus, if $\lambda c_{1}-c_{2} \neq 0$, then we can apply Theorem 2.5 to assure that $c_{1} \mathbf{T}_{1}+c_{2} \mathbf{T}_{2}$ is nonsingular and to find $\left(c_{1} \mathbf{T}_{1}+c_{2} \mathbf{T}_{2}\right)^{-1}$. If $c_{2}=\lambda c_{1}$, then $c_{1} \mathbf{T}+c_{2} \mathbf{T}_{2}$ is nonsingular if and only if $\mathbf{T}+\lambda \mathbf{T}_{2}$ is nonsingular. Now for arbitrary $x, y, z \in \mathbb{C}$ and taking into account that $\mathbf{T}_{1} \mathbf{T}_{2} \mathbf{T}_{2}^{\#}=\lambda \mathbf{T}_{2} \mathbf{T}_{1} \mathbf{T}_{1}^{\#}$, it follows

$$
\left(\mathbf{T}_{1}+\lambda \mathbf{T}_{2}\right)\left(x \mathbf{T}_{1} \mathbf{T}_{1}^{\#}+y \mathbf{T}_{2} \mathbf{T}_{2}^{\#}+z \mathbf{I}_{n}\right)=(x+z) \mathbf{T}_{1}+\lambda(y+z) \mathbf{T}_{2}+\lambda(y+x) \mathbf{T}_{2} \mathbf{T}_{1}^{\#} \mathbf{T}_{1}^{\#}
$$

By solving the following linear system (observe that $\lambda \neq 0$, since otherwise $c_{2}=\lambda c_{1}=0$ )

$$
x+z=1, \quad y+z=\lambda^{-1}, \quad x+y=0,
$$

and

$\mathcal{R}\left[\mathbf{T}_{1}\left(\mathbf{I}_{n}+r_{1} \mathbf{T}_{2}+r_{1} \mathbf{T}_{3}\right)\right]+\mathcal{R}\left[\left(\mathbf{I}_{n}+r_{2} \mathbf{T}_{1}+r_{2} \mathbf{T}_{3}\right) \mathbf{T}_{2}\right]+\mathcal{R}\left[\mathbf{T}_{3}\left(\mathbf{I}_{n}+r_{3} \mathbf{T}_{1}+r_{3} \mathbf{T}_{2}\right)\right]=\mathbb{C}^{n}$

Proof. Let $\alpha_{1}, \alpha_{2}$, and $\alpha_{3}$ denote $r_{1} c_{1}+r_{2} c_{2}, r_{1} c_{1}+r_{3} c_{3}$, and $r_{2} c_{2}+r_{3} c_{3}$, respectively. Moreover, let us take

$$
\mathbf{x} \in \mathcal{N}\left[\mathbf{T}_{1}\left(\mathbf{I}_{n}+r_{1} \mathbf{T}_{2}+r_{1} \mathbf{T}_{3}\right)\right] \cap \mathcal{N}\left[\left(\mathbf{I}_{n}+r_{2} \mathbf{T}_{1}+r_{2} \mathbf{T}_{3}\right) \mathbf{T}_{2}\right] \cap \mathcal{N}\left[\mathbf{T}_{3}\left(\mathbf{I}_{n}+r_{3} \mathbf{T}_{1}+r_{3} \mathbf{T}_{2}\right)\right]
$$

Then, $\mathbf{T}_{1}\left(\mathbf{I}_{n}+r_{1} \mathbf{T}_{2}+r_{1} \mathbf{T}_{3}\right) \mathbf{x}=\left(\mathbf{I}_{n}+r_{2} \mathbf{T}_{1}+r_{2} \mathbf{T}_{3}\right) \mathbf{T}_{2} \mathbf{x}=\mathbf{T}_{3}\left(\mathbf{I}_{n}+r_{3} \mathbf{T}_{1}+r_{3} \mathbf{T}_{2}\right) \mathbf{x}=\mathbf{0}$. Postmultiplying $c_{1} \mathbf{T}_{1}+c_{2} \mathbf{T}_{2}+c_{3} \mathbf{T}_{3}+\alpha_{1} \mathbf{T}_{1} \mathbf{T}_{2}+\alpha_{2} \mathbf{T}_{3} \mathbf{T}_{1}+\alpha_{3} \mathbf{T}_{3} \mathbf{T}_{2}$ by $\mathbf{x}$, it is obtained

$$
\begin{aligned}
& \left(c_{1} \mathbf{T}_{1}+c_{2} \mathbf{T}_{2}+c_{3} \mathbf{T}_{3}+\alpha_{1} \mathbf{T}_{1} \mathbf{T}_{2}+\alpha_{2} \mathbf{T}_{3} \mathbf{T}_{1}+\alpha_{3} \mathbf{T}_{3} \mathbf{T}_{2}\right) \mathbf{x} \\
& =c_{1} \mathbf{T}_{1}\left(\mathbf{I}_{n}+r_{1} \mathbf{T}_{2}+r_{1} \mathbf{T}_{3}\right) \mathbf{x}+c_{2}\left(\mathbf{I}_{n}+r_{2} \mathbf{T}_{1}+r_{2} \mathbf{T}_{3}\right) \mathbf{T}_{2} \mathbf{x}+c_{3} \mathbf{T}_{3}\left(\mathbf{I}_{n}+r_{3} \mathbf{T}_{1}+r_{3} \mathbf{T}_{2}\right) \mathbf{x} \\
& =\mathbf{0}
\end{aligned}
$$

193 which leads to $\mathbf{x}=\mathbf{0}$. So, the proof of (2.26) is complete.

Since $c_{1} \mathbf{T}_{1}+c_{2} \mathbf{T}_{2}+c_{3} \mathbf{T}_{3}+\alpha_{1} \mathbf{T}_{1} \mathbf{T}_{2}+\alpha_{2} \mathbf{T}_{3} \mathbf{T}_{1}+\alpha_{3} \mathbf{T}_{3} \mathbf{T}_{2}$ is nonsingular, then $\bar{c}_{1} \mathbf{T}_{1}^{*}+$ $\bar{c}_{2} \mathbf{T}_{2}^{*}+\bar{c}_{3} \mathbf{T}_{3}^{*}+\bar{\alpha}_{1} \mathbf{T}_{2}^{*} \mathbf{T}_{1}^{*}+\bar{\alpha}_{2} \mathbf{T}_{1}^{*} \mathbf{T}_{3}^{*}+\bar{\alpha}_{3} \mathbf{T}_{2}^{*} \mathbf{T}_{3}^{*}$ is nonsingular. On the other hand, it can be written

$\mathcal{N}\left[\left(\mathbf{I}_{n}+\bar{r}_{3} \mathbf{T}_{1}^{*}+\bar{r}_{3} \mathbf{T}_{2}^{*}\right) \mathbf{T}_{3}^{*}\right] \cap \mathcal{N}\left[\mathbf{T}_{2}^{*}\left(\mathbf{I}_{n}+\bar{r}_{2} \mathbf{T}_{1}^{*}+\bar{r}_{2} \mathbf{T}_{3}^{*}\right)\right] \cap \mathcal{N}\left[\left(\mathbf{I}_{n}+\bar{r}_{1} \mathbf{T}_{2}^{*}+\bar{r}_{1} \mathbf{T}_{3}^{*}\right) \mathbf{T}_{1}^{*}\right]=\{\mathbf{0}\}$.

In view of this equation and [3, pages 74 and 188], it is clearly seen that (2.27) is true. So,

In the following theorem, an expression of the inverse of

$$
c_{1} \mathbf{T}_{1}+c_{2} \mathbf{T}_{2}+c_{3} \mathbf{T}_{3}-c_{4}\left(\mathbf{T}_{1} \mathbf{T}_{2}+\mathbf{T}_{3} \mathbf{T}_{1}+\mathbf{T}_{2} \mathbf{T}_{3}\right)
$$


where $\mathbf{T}_{1}, \mathbf{T}_{2}, \mathbf{T}_{3} \in \mathcal{M}_{n}$ are tripotent matrices, $c_{1}, c_{2}, c_{3} \in \mathbb{C}^{*}$, and $c_{4} \in \mathbb{C}$ is given under some conditions using [10, Theorem 2.5]. It is noteworthy that there is a simple mistake with a minus sign in the formula (2.11) in [10, Theorem 2.5 (ii)]. The corrected form of this formula is

$$
\begin{aligned}
& {\left[\left(c_{1}+c_{2}\right)^{2}-c_{3}^{2}\right]\left(c_{1} \mathbf{T}_{1}+c_{2} \mathbf{T}_{2}-c_{3} \mathbf{T}_{1} \mathbf{T}_{2}\right)^{-1}} \\
& \quad=\left(c_{1}+c_{2}\right) \mathbf{T}_{2}+c_{3} \mathbf{T}_{2} \mathbf{T}_{1}+c_{2}^{-1}\left(c_{1}^{2}+c_{1} c_{2}-c_{3}^{2}\right)\left(\mathbf{T}_{2}-\mathbf{T}_{2} \mathbf{T}_{1}^{2}\right) .
\end{aligned}
$$

Of course, this expression is used in the foregoing theorem.

Theorem 2.7. Let $c_{1}, c_{2}, c_{3} \in \mathbb{C}^{*}, c_{4} \in \mathbb{C}, \mathbf{T}_{1}, \mathbf{T}_{2}$, and $\mathbf{T}_{3} \in \mathcal{M}_{n}$ be nonzero tripotent matrices such that $\mathbf{T}_{1}^{2} \mathbf{T}_{2}-\mathbf{T}_{2}^{2} \mathbf{T}_{1}=\mathbf{T}_{2}^{2} \mathbf{T}_{3}+\mathbf{T}_{3}^{2} \mathbf{T}_{2}=\mathbf{T}_{1}^{2} \mathbf{T}_{3}-\mathbf{T}_{3}^{2} \mathbf{T}_{1}=\mathbf{0}$ and let us say, for the sake of simplicity, $\alpha=\left(c_{1}+c_{3}\right)^{2}-c_{4}^{2}, \beta=\left(c_{1}+c_{2}\right)^{2}-c_{4}^{2}, \gamma=\left(c_{2}-c_{3}\right)^{2}-c_{4}^{2}$,

$$
\mathbf{T}_{-}=c_{1} \mathbf{T}_{1}+c_{2} \mathbf{T}_{2}+c_{3} \mathbf{T}_{3}-c_{4}\left(\mathbf{T}_{1} \mathbf{T}_{2}+\mathbf{T}_{3} \mathbf{T}_{1}+\mathbf{T}_{2} \mathbf{T}_{3}\right),
$$

and

$$
\mathbf{T}_{+}=c_{1} \mathbf{T}_{1}+c_{2} \mathbf{T}_{2}+c_{3} \mathbf{T}_{3}+c_{4}\left(\mathbf{T}_{1} \mathbf{T}_{2}+\mathbf{T}_{3} \mathbf{T}_{1}+\mathbf{T}_{2} \mathbf{T}_{3}\right) .
$$

(i) Let $\mathbf{T}_{1}$ be nonsingular and $\alpha \neq 0$. If $\beta=0$, then $\mathbf{T}_{-}$or $\mathbf{T}_{+}$is singular. If $\beta \neq 0$, then $\mathbf{T}_{-}$is nonsingular and

$$
\begin{aligned}
& \alpha \beta \mathbf{T}_{-}^{-1} \\
& =\alpha\left[\left(c_{1}+c_{2}\right) \mathbf{T}_{1} \mathbf{T}_{2}^{2}+c_{4} \mathbf{T}_{1} \mathbf{T}_{2}+\frac{c_{4}}{c_{1}}\left(c_{1}+c_{2}\right)\left(\mathbf{T}_{2}^{2}-\mathbf{T}_{1} \mathbf{T}_{2}\right)+\frac{c_{4}^{2}}{c_{1}}\left(\mathbf{T}_{2}-\mathbf{T}_{1} \mathbf{T}_{2}^{2}\right)\right] \\
& \quad+\beta\left[c_{4} \mathbf{T}_{1} \mathbf{T}_{3}+\frac{\alpha}{c_{1}}\left(\mathbf{T}_{1}-\mathbf{T}_{1} \mathbf{T}_{2}^{2}-\mathbf{T}_{1} \mathbf{T}_{3}^{2}\right)+\left(c_{1}+c_{3}\right) \mathbf{T}_{1} \mathbf{T}_{3}^{2}\right] .
\end{aligned}
$$

(ii) Let $\mathbf{T}_{2}$ be nonsingular and $\beta \neq 0$. If $\gamma=0$, then $\mathbf{T}_{-}$or $\mathbf{T}_{+}$is singular. If $\gamma \neq 0$, then $\mathbf{T}_{-}$is nonsingular and

$$
\begin{aligned}
& \beta \gamma \mathbf{T}_{-}^{-1} \\
& =\beta\left[\left(c_{2}-c_{3}\right) \mathbf{T}_{2} \mathbf{T}_{3}^{2}+c_{4} \mathbf{T}_{2} \mathbf{T}_{3}+\frac{c_{4}}{c_{2}}\left(c_{2}-c_{3}\right)\left(\mathbf{T}_{3}^{2}+\mathbf{T}_{2} \mathbf{T}_{3}\right)-\frac{c_{4}^{2}}{c_{2}}\left(\mathbf{T}_{3}+\mathbf{T}_{2} \mathbf{T}_{3}^{2}\right)\right] \\
& \quad+\gamma\left[c_{4} \mathbf{T}_{2} \mathbf{T}_{1}+\frac{\beta}{c_{2}}\left(\mathbf{T}_{2}-\mathbf{T}_{2} \mathbf{T}_{3}^{2}-\mathbf{T}_{2} \mathbf{T}_{1}^{2}\right)+\left(c_{1}+c_{2}\right) \mathbf{T}_{2} \mathbf{T}_{1}^{2}\right] .
\end{aligned}
$$

(iii) Let $\mathbf{T}_{3}$ be nonsingular and $\alpha \neq 0$. If $\gamma=0$, then $\mathbf{T}_{-}$or $\mathbf{T}_{+}$is singular. If $\gamma \neq 0$, then $\mathbf{T}_{-}$is nonsingular and

$$
\begin{aligned}
& \alpha \gamma \mathbf{T}_{-}^{-1}=\alpha\left[\left(c_{3}-c_{2}\right) \mathbf{T}_{3} \mathbf{T}_{2}^{2}+c_{4} \mathbf{T}_{3} \mathbf{T}_{2}\right] \\
& \quad+\frac{\gamma}{c_{3}}\left[\alpha\left(\mathbf{T}_{3}-\mathbf{T}_{3} \mathbf{T}_{2}^{2}\right)+c_{4}\left(c_{1}+c_{3}\right) \mathbf{T}_{1}^{2}-c_{1} c_{4} \mathbf{T}_{3} \mathbf{T}_{1}-c_{1}\left(c_{1}+c_{3}\right) \mathbf{T}_{3} \mathbf{T}_{1}^{2}+c_{4}^{2} \mathbf{T}_{1}\right] .
\end{aligned}
$$

Proof. First, let us prove the following claim:

Claim: Let $\mathbf{X}, \mathbf{Y}, \mathbf{Z} \in \mathcal{M}_{n}$ be nonzero tripotent matrices such that $\mathbf{X}$ is nonsingular and

$$
\mathbf{Y}=\mathbf{Y}^{2} \mathbf{X}, \quad \mathbf{Y}^{2} \mathbf{Z}+\mathbf{Z}^{2} \mathbf{Y}=\mathbf{0}, \quad \mathbf{Z}=\mathbf{Z}^{2} \mathbf{X} .
$$

Then $\mathbf{X}, \mathbf{Y}, \mathbf{Z}$ can be represented as follows:

$$
\mathbf{X}=\mathbf{S}\left(\begin{array}{cc}
\mathbf{A} & \mathbf{0} \\
\mathbf{D} & \mathbf{E}
\end{array}\right) \mathbf{S}^{-1}, \quad \mathbf{Y}=\mathbf{S}\left(\begin{array}{cc}
\mathbf{A} & \mathbf{0} \\
\mathbf{0} & \mathbf{0}
\end{array}\right) \mathbf{S}^{-1}, \quad \mathbf{Z}=\mathbf{S}\left(\begin{array}{cc}
\mathbf{0} & \mathbf{0} \\
\mathbf{0} & \mathbf{K}
\end{array}\right) \mathbf{S}^{-1},
$$

where $\mathbf{S} \in \mathcal{M}_{n}$ is nonsingular, $\mathbf{A} \in \mathcal{M}_{r}, \mathbf{K} \in \mathcal{M}_{n-r}$, and

$$
\mathbf{K D}=\mathbf{0}, \quad \mathbf{K}^{2} \mathbf{E}=\mathbf{K}, \quad \mathbf{A}^{2}=\mathbf{I}_{r}, \quad \mathbf{E}^{2}=\mathbf{I}_{n-r}, \quad \mathbf{D A}=-\mathbf{E D} .
$$


Proof of the claim. Since $\mathbf{Y}$ is tripotent, there exists a nonsingular $\mathbf{S} \in M_{n}$ such that $\mathbf{Y}=$ $\mathbf{S}(\mathbf{A} \oplus \mathbf{0}) \mathbf{S}^{-1}$, where $\mathbf{A} \in \mathcal{M}_{r}$ and $r=\operatorname{rank}(\mathbf{A})$. Since $\mathbf{A}$ is nonsingular and $\mathbf{Y}^{3}=\mathbf{Y}$, we have $\mathbf{A}^{2}=\mathbf{I}_{r}$. Let us write

$$
\mathbf{X}=\mathbf{S}\left(\begin{array}{ll}
\mathbf{B} & \mathbf{C} \\
\mathbf{D} & \mathbf{E}
\end{array}\right) \mathbf{S}^{-1}, \quad \mathbf{Z}=\mathbf{S}\left(\begin{array}{cc}
\mathbf{F} & \mathbf{G} \\
\mathbf{H} & \mathbf{K}
\end{array}\right) \mathbf{S}^{-1}, \quad \mathbf{B}, \mathbf{F} \in \mathcal{M}_{r} .
$$

From the first equality of (2.31) it follows that

$$
\mathbf{B}=\mathbf{A}, \quad \mathbf{C}=\mathbf{0} .
$$

The middle equality of (2.31) together with $\mathbf{A}^{2}=\mathbf{I}_{r}$ lead to

$$
\mathbf{F}^{2} \mathbf{A}+\mathbf{F}=\mathbf{0}, \quad \mathbf{G}=\mathbf{0}, \quad \mathbf{H F}+\mathbf{K H}=\mathbf{0} .
$$

The last equality of (2.31) in conjunction with (2.34), $\mathbf{G}=\mathbf{0}$, and $\mathbf{H F}+\mathbf{K H}=\mathbf{0}$ yield

$$
\mathbf{F}=\mathbf{F}^{2} \mathbf{A}, \quad \mathbf{H}=\mathbf{K}^{2} \mathbf{D}, \quad \mathbf{K}=\mathbf{K}^{2} \mathbf{E} .
$$

The first equalities of (2.35) and (2.36) imply $\mathbf{F}=\mathbf{0}$. Premultiplying by $\mathbf{Z}$ the second equality of (2.31) and using the tripotency of $\mathbf{T}_{3}$ lead to $\mathbf{Z} \mathbf{Y}^{2} \mathbf{Z}+\mathbf{Z Y}=\mathbf{0}$, and this latter equality yields $\mathbf{H A}=\mathbf{0}$, and having in mind the nonsingularity of $\mathbf{A}$ we can deduce $\mathbf{H}=\mathbf{0}$. Thus, the representations given in (2.32) are proven.

Furthermore, the tripotency of $\mathbf{Z}$ and $\mathbf{G}=\mathbf{0}$ imply $\mathbf{K}^{3}=\mathbf{K}$, and thus, from the second equality of (2.36) it follows that $\mathbf{K D}=\mathbf{0}$. Thus we have proved the first equality of (2.33). The second equality of (2.33) was deduced in (2.36), while the remaining equalities of (2.33) follow from $\mathbf{X}^{2}=\mathbf{I}_{n}$.

(i) Let us assume that $\mathbf{T}_{1}$ is nonsingular and $\alpha \neq 0$. The condition $\mathbf{T}_{1}^{2} \mathbf{T}_{2}-\mathbf{T}_{2}^{2} \mathbf{T}_{1}=$ $\mathbf{T}_{2}^{2} \mathbf{T}_{3}+\mathbf{T}_{3}^{2} \mathbf{T}_{2}=\mathbf{T}_{1}^{2} \mathbf{T}_{3}-\mathbf{T}_{3}^{2} \mathbf{T}_{1}=\mathbf{0}$ turns into

$$
\mathbf{T}_{2}=\mathbf{T}_{2}^{2} \mathbf{T}_{1}, \quad \mathbf{T}_{2}^{2} \mathbf{T}_{3}+\mathbf{T}_{3}^{2} \mathbf{T}_{2}=\mathbf{0}, \quad \mathbf{T}_{3}=\mathbf{T}_{3}^{2} \mathbf{T}_{1}
$$

222 since $\mathbf{T}_{1}^{2}=\mathbf{I}_{n}$. By applying the claim, we can write

$$
\mathbf{T}_{1}=\mathbf{S}\left(\begin{array}{cc}
\mathbf{A} & \mathbf{0} \\
\mathbf{D} & \mathbf{E}
\end{array}\right) \mathbf{S}^{-1}, \quad \mathbf{T}_{2}=\mathbf{S}\left(\begin{array}{cc}
\mathbf{A} & \mathbf{0} \\
\mathbf{0} & \mathbf{0}
\end{array}\right) \mathbf{S}^{-1}, \quad \mathbf{T}_{3}=\mathbf{S}\left(\begin{array}{cc}
\mathbf{0} & \mathbf{0} \\
\mathbf{0} & \mathbf{K}
\end{array}\right) \mathbf{S}^{-1},
$$

and in addition, the relations (2.33) hold. Observe that $\mathbf{K}$ must be a nonzero tripotent matrix since $\mathbf{T}_{3}$ is nonzero and tripotent. On the other hand, using (2.37), it can be written

$$
\mathbf{T}_{-}=\mathbf{S}\left(\begin{array}{cc}
\left(c_{1}+c_{2}\right) \mathbf{A}-c_{4} \mathbf{I}_{r} & \mathbf{0} \\
c_{1} \mathbf{D}-c_{4} \mathbf{D A} & c_{3} \mathbf{K}+c_{1} \mathbf{E}-c_{4} \mathbf{K E}
\end{array}\right) \mathbf{S}^{-1} .
$$

According to [10, Theorem 2.5 (ii)], the matrix $c_{3} \mathbf{K}+c_{1} \mathbf{E}-c_{4} \mathbf{K E}$ is nonsingular and

$$
\left(c_{3} \mathbf{K}+c_{1} \mathbf{E}-c_{4} \mathbf{K E}\right)^{-1}=\alpha^{-1}\left[\left(c_{1}+c_{3}\right) \mathbf{E}+c_{4} \mathbf{E K}+c_{1}^{-1}\left(c_{3}^{2}+c_{3} c_{1}-c_{4}^{2}\right)\left(\mathbf{E}-\mathbf{E K}^{2}\right)\right],
$$

which having in mind $\alpha=\left(c_{1}+c_{3}\right)^{2}-c_{4}^{2}$, becomes to

$$
\left(c_{3} \mathbf{K}+c_{1} \mathbf{E}-c_{4} \mathbf{K E}\right)^{-1}=\alpha^{-1}\left[c_{4} \mathbf{E K}+\alpha c_{1}^{-1}\left(\mathbf{E}-\mathbf{E K}^{2}\right)+\left(c_{1}+c_{3}\right) \mathbf{E K}^{2}\right] .
$$

From (2.38) it is obtained that $\mathbf{T}_{-}^{-1}$ is nonsingular if and only if $\left(c_{1}+c_{2}\right) \mathbf{A}-c_{4} \mathbf{I}_{r}$ is nonsingular (recall that the first row in the block matrix appearing in (2.38) must be present, since otherwise, $\mathbf{T}_{2}=\mathbf{0}$ ). The following equality is evident:

$$
\left[\left(c_{1}+c_{2}\right) \mathbf{A}-c_{4} \mathbf{I}_{r}\right]\left[\left(c_{1}+c_{2}\right) \mathbf{A}+c_{4} \mathbf{I}_{r}\right]=\beta \mathbf{I}_{r},
$$

If $\beta=0$, then (2.40) implies that $\left(c_{1}+c_{2}\right) \mathbf{A}-c_{4} \mathbf{I}_{r}$ or $\left(c_{1}+c_{2}\right) \mathbf{A}+c_{4} \mathbf{I}_{r}$ is singular. Hence $\mathbf{T}_{-}$or $\mathbf{T}_{+}$is singular by (2.38). 


$$
\mathbf{M}=-\left[c_{3} \mathbf{K}+c_{1} \mathbf{E}-c_{4} \mathbf{K E}\right]^{-1}\left(c_{1} \mathbf{D}-c_{4} \mathbf{D A}\right)\left[\left(c_{1}+c_{2}\right) \mathbf{A}-c_{4} \mathbf{I}_{r}\right]^{-1} .
$$

$$
\left[c_{3} \mathbf{K}+c_{1} \mathbf{E}-c_{4} \mathbf{K E}\right]^{-1}\left(c_{1} \mathbf{D}-c_{4} \mathbf{D A}\right)=\mathbf{E D}+c_{1}^{-1} c_{4} \mathbf{D}
$$

${ }_{235}$ By using (2.33), (2.41), and (2.44), the matrix $\mathbf{M}$ defined in (2.43) can be simplified:

$$
\begin{aligned}
\mathbf{M} & =-\beta^{-1}\left[\mathbf{E D}+c_{1}^{-1} c_{4} \mathbf{D}\right]\left[\left(c_{1}+c_{2}\right) \mathbf{A}+c_{4} \mathbf{I}_{r}\right] \\
& =-\beta^{-1}\left[\left(c_{1}+c_{2}\right) \mathbf{E D A}+c_{4} \mathbf{E D}+c_{1}^{-1} c_{4}\left(c_{1}+c_{2}\right) \mathbf{D A}+c_{1}^{-1} c_{4}^{2} \mathbf{D}\right] \\
& =-\beta^{-1}\left[\left(c_{1}^{-1} c_{4}^{2}-c_{1}-c_{2}\right) \mathbf{D}+c_{1}^{-1} c_{4} c_{2} \mathbf{D A}\right] .
\end{aligned}
$$

${ }_{236}$ Combining (2.39), (2.41), (2.42), and (2.45), it is obtained

$$
\begin{aligned}
& \alpha \beta \mathbf{T}_{-}^{-1}=\mathbf{S}\left\{\alpha \left[\left(c_{1}+c_{2}\right)\left(\begin{array}{cc}
\mathbf{A} & \mathbf{0} \\
\mathbf{D} & \mathbf{0}
\end{array}\right)+c_{4}\left(\begin{array}{cc}
\mathbf{I}_{r} & \mathbf{0} \\
-\mathbf{E D} & \mathbf{0}
\end{array}\right)\right.\right. \\
& \left.+c_{1}^{-1} c_{4}\left(c_{1}+c_{2}\right)\left(\begin{array}{cc}
\mathbf{0} & \mathbf{0} \\
\mathbf{E D} & \mathbf{0}
\end{array}\right)+c_{1}^{-1} c_{4}^{2}\left(\begin{array}{cc}
\mathbf{0} & \mathbf{0} \\
-\mathbf{D} & \mathbf{0}
\end{array}\right)\right] \\
& +\beta\left[c_{4}\left(\begin{array}{cc}
\mathbf{0} & \mathbf{0} \\
\mathbf{0} & \mathbf{E K}
\end{array}\right)+\alpha c_{1}^{-1}\left(\begin{array}{cc}
\mathbf{0} & \mathbf{0} \\
\mathbf{0} & \mathbf{E}-\mathbf{E K}^{2}
\end{array}\right)\right. \\
& \left.\left.+\left(c_{1}+c_{3}\right)\left(\begin{array}{cc}
\mathbf{0} & \mathbf{0} \\
\mathbf{0} & \mathbf{E K}^{2}
\end{array}\right)\right]\right\} \mathbf{S}^{-1} .
\end{aligned}
$$

Then, considering the following equalities in (2.46)

$$
\begin{gathered}
\mathbf{T}_{1} \mathbf{T}_{3}=\mathbf{S}\left(\begin{array}{cc}
\mathbf{0} & \mathbf{0} \\
\mathbf{0} & \mathbf{E K}
\end{array}\right) \mathbf{S}^{-1}, \quad \mathbf{T}_{1} \mathbf{T}_{3}^{2}=\mathbf{S}\left(\begin{array}{cc}
\mathbf{0} & \mathbf{0} \\
\mathbf{0} & \mathbf{E K}^{2}
\end{array}\right) \mathbf{S}^{-1}, \\
\mathbf{T}_{2}^{2}-\mathbf{T}_{1} \mathbf{T}_{2}=\mathbf{S}\left(\begin{array}{cc}
\mathbf{0} & \mathbf{0} \\
\mathbf{E D} & \mathbf{0}
\end{array}\right) \mathbf{S}^{-1}, \quad \mathbf{T}_{1} \mathbf{T}_{2}^{2}=\mathbf{S}\left(\begin{array}{cc}
\mathbf{A} & \mathbf{0} \\
\mathbf{D} & \mathbf{0}
\end{array}\right) \mathbf{S}^{-1}, \\
\mathbf{T}_{1}-\mathbf{T}_{1} \mathbf{T}_{2}^{2}-\mathbf{T}_{1} \mathbf{T}_{3}^{2}=\mathbf{S}\left(\begin{array}{cc}
\mathbf{0} & \mathbf{0} \\
\mathbf{0} & \mathbf{E}-\mathbf{E} \mathbf{K}^{2}
\end{array}\right) \mathbf{S}^{-1},
\end{gathered}
$$

and

$$
\mathbf{T}_{1} \mathbf{T}_{2}=\mathbf{S}\left(\begin{array}{cc}
\mathbf{I}_{r} & \mathbf{0} \\
-\mathbf{E D} & \mathbf{0}
\end{array}\right) \mathbf{S}^{-1}, \quad \mathbf{T}_{2}-\mathbf{T}_{1} \mathbf{T}_{2}^{2}=\mathbf{S}\left(\begin{array}{cc}
\mathbf{0} & \mathbf{0} \\
-\mathbf{D} & \mathbf{0}
\end{array}\right) \mathbf{S}^{-1}
$$

${ }_{237}$ leads to the formula (2.28). So the proof of part (i) is complete.

(ii) Let us assume that $\mathbf{T}_{2}$ is nonsingular and $\beta \neq 0$. The condition $\mathbf{T}_{1}^{2} \mathbf{T}_{2}-\mathbf{T}_{2}^{2} \mathbf{T}_{1}=$ ${ }^{239} \quad \mathbf{T}_{2}^{2} \mathbf{T}_{3}+\mathbf{T}_{3}^{2} \mathbf{T}_{2}=\mathbf{T}_{1}^{2} \mathbf{T}_{3}-\mathbf{T}_{3}^{2} \mathbf{T}_{1}=\mathbf{0}$ turns into

$$
\mathbf{T}_{1}^{2} \mathbf{T}_{2}=\mathbf{T}_{1}, \quad \mathbf{T}_{3}+\mathbf{T}_{3}^{2} \mathbf{T}_{2}=\mathbf{0}, \quad \mathbf{T}_{1}^{2} \mathbf{T}_{3}=\mathbf{T}_{3}^{2} \mathbf{T}_{1}
$$
since $\mathbf{T}_{2}^{2}=\mathbf{I}_{n}$. We can apply the claim for $\mathbf{X}=-\mathbf{T}_{2}, \mathbf{Y}=\mathbf{T}_{3}$, and $\mathbf{Z}=-\mathbf{T}_{1}$ obtaining 


$$
\mathbf{M}=-\left[c_{1} \mathbf{K}+c_{2} \mathbf{E}-c_{4} \mathbf{K E}\right]^{-1}\left(c_{2} \mathbf{D}-c_{4} \mathbf{D A}\right)\left[\left(-c_{2}+c_{3}\right) \mathbf{A}+c_{4} \mathbf{I}_{r}\right]^{-1} .
$$

By the first equality of (2.49) and (2.51)

$$
\left[c_{1} \mathbf{K}+c_{2} \mathbf{E}-c_{4} \mathbf{K E}\right]^{-1}\left(c_{2} \mathbf{D}-c_{4} \mathbf{D A}\right)=\mathbf{E D}-c_{2}^{-1} c_{4} \mathbf{D} .
$$

${ }_{254}$ By doing some elementary algebra and using (2.49 and (2.53) we can simplify $\mathbf{M}$ obtaining

$$
\mathbf{M}=\gamma^{-1}\left[\left(c_{2}-c_{3}-c_{2}^{-1} c_{4}^{2}\right) \mathbf{D}+c_{2}^{-1} c_{3} c_{4} \mathbf{D A}\right] .
$$

Combining (2.51), (2.53), (2.54), and (2.55) it is obtained

$$
\begin{aligned}
& \beta \gamma \mathbf{T}_{-}^{-1}=\mathbf{S}\left\{\beta \left[\left(-c_{2}+c_{3}\right)\left(\begin{array}{cc}
\mathbf{A} & \mathbf{0} \\
-\mathbf{D} & \mathbf{0}
\end{array}\right)+c_{4}\left(\begin{array}{cc}
-\mathbf{I}_{r} & \mathbf{0} \\
\mathbf{E D} & \mathbf{0}
\end{array}\right)\right.\right. \\
& \left.+c_{2}^{-1} c_{4}\left(-c_{2}+c_{3}\right)\left(\begin{array}{cc}
\mathbf{0} & \mathbf{0} \\
\mathbf{E D} & \mathbf{0}
\end{array}\right)-c_{2}^{-1} c_{4}^{2}\left(\begin{array}{cc}
\mathbf{0} & \mathbf{0} \\
\mathbf{D} & \mathbf{0}
\end{array}\right)\right] \\
& +\gamma\left[c_{4}\left(\begin{array}{cc}
\mathbf{0} & \mathbf{0} \\
\mathbf{0} & \mathbf{E K}
\end{array}\right)+\beta c_{2}^{-1}\left(\begin{array}{cc}
\mathbf{0} & \mathbf{0} \\
\mathbf{0} & \mathbf{E}-\mathbf{E K}^{2}
\end{array}\right)\right. \\
& \left.\left.+\left(c_{1}+c_{2}\right)\left(\begin{array}{cc}
\mathbf{0} & \mathbf{0} \\
\mathbf{0} & \mathbf{E K}^{2}
\end{array}\right)\right]\right\} \mathbf{S}^{-1} .
\end{aligned}
$$

On the other hand, the following equalities can be written:

$$
\mathbf{T}_{2} \mathbf{T}_{3}=\mathbf{S}\left(\begin{array}{cc}
-\mathbf{I}_{r} & \mathbf{0} \\
\mathbf{E D} & \mathbf{0}
\end{array}\right) \mathbf{S}^{-1}, \quad \mathbf{T}_{2} \mathbf{T}_{1}^{2}=\mathbf{S}\left(\begin{array}{cc}
\mathbf{0} & \mathbf{0} \\
\mathbf{0} & \mathbf{E K}^{2}
\end{array}\right) \mathbf{S}^{-1},
$$




$$
\begin{aligned}
& \mathbf{T}_{3}^{2}+\mathbf{T}_{2} \mathbf{T}_{3}=\mathbf{S}\left(\begin{array}{cc}
\mathbf{0} & \mathbf{0} \\
\mathbf{E D} & \mathbf{0}
\end{array}\right) \mathbf{S}^{-1}, \quad \mathbf{T}_{2} \mathbf{T}_{1}=\mathbf{S}\left(\begin{array}{cc}
\mathbf{0} & \mathbf{0} \\
\mathbf{0} & \mathbf{E K}
\end{array}\right) \mathbf{S}^{-1} \\
& \mathbf{T}_{2} \mathbf{T}_{3}^{2}=\mathbf{S}\left(\begin{array}{cc}
-\mathbf{A} & \mathbf{0} \\
\mathbf{D} & \mathbf{0}
\end{array}\right) \mathbf{S}^{-1}, \quad \mathbf{T}_{3}+\mathbf{T}_{2} \mathbf{T}_{3}^{2}=\mathbf{S}\left(\begin{array}{cc}
\mathbf{0} & \mathbf{0} \\
\mathbf{D} & \mathbf{0}
\end{array}\right) \mathbf{S}^{-1}
\end{aligned}
$$

and

$$
\mathbf{T}_{2}-\mathbf{T}_{2} \mathbf{T}_{3}^{2}-\mathbf{T}_{2} \mathbf{T}_{1}^{2}=\mathbf{S}\left(\begin{array}{cc}
\mathbf{0} & \mathbf{0} \\
\mathbf{0} & \mathbf{E}-\mathbf{E} \mathbf{K}^{2}
\end{array}\right) \mathbf{S}^{-1}
$$

Substituting these equalities in (2.56) leads to the formula (2.29) which is the desired result.

(iii) Let us assume that $\mathbf{T}_{3}$ is nonsingular and $\alpha \neq 0$. The condition $\mathbf{T}_{1}^{2} \mathbf{T}_{2}-\mathbf{T}_{2}^{2} \mathbf{T}_{1}=$ $\mathbf{T}_{2}^{2} \mathbf{T}_{3}+\mathbf{T}_{3}^{2} \mathbf{T}_{2}=\mathbf{T}_{1}^{2} \mathbf{T}_{3}-\mathbf{T}_{3}^{2} \mathbf{T}_{1}=\mathbf{0}$ turns into

$$
\mathbf{T}_{1}^{2} \mathbf{T}_{2}=\mathbf{T}_{2}^{2} \mathbf{T}_{1}, \quad \mathbf{T}_{2}^{2} \mathbf{T}_{3}+\mathbf{T}_{2}=\mathbf{0}, \quad \mathbf{T}_{1}^{2} \mathbf{T}_{3}=\mathbf{T}_{1}
$$

since $\mathbf{T}_{3}^{2}=\mathbf{I}_{n}$. By applying the claim for $\mathbf{X}=\mathbf{T}_{3}, \mathbf{Y}=-\mathbf{T}_{2}$, and $\mathbf{Z}=\mathbf{T}_{1}$, we can write

$$
\mathbf{T}_{1}=\mathbf{S}\left(\begin{array}{cc}
\mathbf{0} & \mathbf{0} \\
\mathbf{0} & \mathbf{K}
\end{array}\right) \mathbf{S}^{-1}, \quad \mathbf{T}_{2}=\mathbf{S}\left(\begin{array}{cc}
-\mathbf{A} & \mathbf{0} \\
\mathbf{0} & \mathbf{0}
\end{array}\right) \mathbf{S}^{-1}, \quad \mathbf{T}_{3}=\mathbf{S}\left(\begin{array}{cc}
\mathbf{A} & \mathbf{0} \\
\mathbf{D} & \mathbf{E}
\end{array}\right) \mathbf{S}^{-1}
$$

where $\mathbf{S} \in \mathcal{M}_{n}$ is nonsingular, $\mathbf{A} \in \mathcal{M}_{r}, \mathbf{K} \in \mathcal{M}_{n-r}$, and blocks $\mathbf{A}, \mathbf{D}, \mathbf{E}, \mathbf{K}$ satisfy (2.33). Using (2.57), it can be written

$$
\mathbf{T}_{-}=\mathbf{S}\left(\begin{array}{cc}
\left(-c_{2}+c_{3}\right) \mathbf{A}+c_{4} \mathbf{I}_{r} & \mathbf{0} \\
c_{3} \mathbf{D} & c_{3} \mathbf{E}+c_{1} \mathbf{K}-c_{4} \mathbf{E K}
\end{array}\right) \mathbf{S}^{-1} .
$$

Observe that $\mathbf{K} \neq \mathbf{0}$, since otherwise $\mathbf{T}_{1}=\mathbf{0}$. Also, $\mathbf{E}$ is nonsingular because $\mathbf{T}_{3}$ is nonsingular. According to [10, Thorem 2.5 (i)], the matrix $c_{3} \mathbf{E}+c_{1} \mathbf{K}-c_{4} \mathbf{E K}$ is nonsingular and

$$
\begin{aligned}
& \left(c_{3} \mathbf{E}+c_{1} \mathbf{K}-c_{4} \mathbf{E K}\right)^{-1} \\
& \quad=\alpha^{-1} c_{3}^{-1}\left[\alpha \mathbf{E}+c_{4}\left(c_{3}+c_{1}\right) \mathbf{K}^{2}-c_{1} c_{4} \mathbf{E K}-c_{1}\left(c_{3}+c_{1}\right) \mathbf{E K}^{2}+c_{4}^{2} \mathbf{K}\right] .
\end{aligned}
$$

From (2.58), it is obtained that $\mathbf{T}_{-}$is nonsingular if and only if $\left(-c_{2}+c_{3}\right) \mathbf{A}+c_{4} \mathbf{I}_{r}$ is nonsingular. It is evident that

$$
\left[\left(-c_{2}+c_{3}\right) \mathbf{A}+c_{4} \mathbf{I}_{r}\right]\left[\left(-c_{2}+c_{3}\right) \mathbf{A}-c_{4} \mathbf{I}_{r}\right]=\gamma \mathbf{I}_{r} .
$$

If $\gamma=0$, then (2.53) yields that $\left(-c_{2}+c_{3}\right) \mathbf{A}+c_{4} \mathbf{I}_{r}$ or $\left(-c_{2}+c_{3}\right) \mathbf{A}-c_{4} \mathbf{I}_{r}$ is singular. Hence $\mathbf{T}_{-}$or $\mathbf{T}_{+}$is singular, by (2.58).

Now, let $\gamma \neq 0$. From (2.60) the matrix $\left(-c_{2}+c_{3}\right) \mathbf{A}+c_{4} \mathbf{I}_{r}$ is nonsingular and

$$
\left[\left(-c_{2}+c_{3}\right) \mathbf{A}+c_{4} \mathbf{I}_{r}\right]^{-1}=\gamma^{-1}\left[\left(-c_{2}+c_{3}\right) \mathbf{A}-c_{4} \mathbf{I}_{r}\right] .
$$

Using [18, Problem 19 (c)], the inverse of matrix in (2.58) is obtained as

$$
\mathbf{T}_{-}^{-1}=\mathbf{S}\left(\begin{array}{cc}
{\left[\left(-c_{2}+c_{3}\right) \mathbf{A}+c_{4} \mathbf{I}_{r}\right]^{-1}} & \mathbf{0} \\
\mathbf{M} & {\left[c_{3} \mathbf{E}+c_{1} \mathbf{K}-c_{4} \mathbf{E K}\right]^{-1}}
\end{array}\right) \mathbf{S}^{-1},
$$

where

$$
\mathbf{M}=-\left[c_{3} \mathbf{E}+c_{1} \mathbf{K}-c_{4} \mathbf{E K}\right]^{-1} c_{3} \mathbf{D}\left[\left(-c_{2}+c_{3}\right) \mathbf{A}+c_{4} \mathbf{I}_{r}\right]^{-1} .
$$

Since $\mathbf{K}$ and $\mathbf{D}$ satisfy (2.33), then (2.59) implies $\left[c_{3} \mathbf{E}+c_{1} \mathbf{K}-c_{4} \mathbf{E K}\right]^{-1} \mathbf{D}=c_{3}^{-1} \mathbf{E D}$. Therefore, (2.60) and (2.33) lead to

$$
\mathbf{M}=-\gamma^{-1} \mathbf{E D}\left[\left(-c_{2}+c_{3}\right) \mathbf{A}-c_{4} \mathbf{I}_{r}\right]=\gamma^{-1}\left[\left(-c_{2}+c_{3}\right) \mathbf{D}-c_{4} \mathbf{E D}\right] .
$$




$$
\begin{aligned}
\alpha \gamma \mathbf{T}_{-}^{-1}=\mathbf{S}\left\{\alpha\left[\left(-c_{2}+c_{3}\right)\left(\begin{array}{cc}
\mathbf{A} & \mathbf{0} \\
\mathbf{D} & \mathbf{0}
\end{array}\right)+c_{4}\left(\begin{array}{cc}
-\mathbf{I}_{r} & \mathbf{0} \\
\mathbf{E D} & \mathbf{0}
\end{array}\right)\right]\right. \\
+\gamma c_{3}^{-1}\left[\alpha\left(\begin{array}{cc}
\mathbf{0} & \mathbf{0} \\
\mathbf{0} & \mathbf{E}
\end{array}\right)+c_{4}\left(c_{1}+c_{3}\right)\left(\begin{array}{cc}
\mathbf{0} & \mathbf{0} \\
\mathbf{0} & \mathbf{K}^{2}
\end{array}\right)-c_{1} c_{4}\left(\begin{array}{cc}
\mathbf{0} & \mathbf{0} \\
\mathbf{0} & \mathbf{E K}
\end{array}\right)\right. \\
\left.\left.-c_{1}\left(c_{1}+c_{3}\right)\left(\begin{array}{cc}
\mathbf{0} & \mathbf{0} \\
\mathbf{0} & \mathbf{E K}^{2}
\end{array}\right)+c_{4}^{2}\left(\begin{array}{cc}
\mathbf{0} & \mathbf{0} \\
\mathbf{0} & \mathbf{K}
\end{array}\right)\right]\right\} \mathbf{S}^{-1}
\end{aligned}
$$

On the other hand, by employing (2.57) and the relations given in (2.33), the following equalities can be written

$$
\begin{array}{ll}
\mathbf{T}_{3} \mathbf{T}_{1}^{2}=\mathbf{S}\left(\begin{array}{cc}
\mathbf{0} & \mathbf{0} \\
\mathbf{0} & \mathbf{E K}^{2}
\end{array}\right) \mathbf{S}^{-1}, & \mathbf{T}_{1}^{2}=\mathbf{S}\left(\begin{array}{cc}
\mathbf{0} & \mathbf{0} \\
\mathbf{0} & \mathbf{K}^{2}
\end{array}\right) \mathbf{S}^{-1} \\
\mathbf{T}_{3} \mathbf{T}_{2}=\mathbf{S}\left(\begin{array}{cc}
-\mathbf{I}_{r} & \mathbf{0} \\
\mathbf{E D} & \mathbf{0}
\end{array}\right) \mathbf{S}^{-1}, & \mathbf{T}_{3} \mathbf{T}_{2}^{2}=\mathbf{S}\left(\begin{array}{cc}
\mathbf{A} & \mathbf{0} \\
\mathbf{D} & \mathbf{0}
\end{array}\right) \mathbf{S}^{-1},
\end{array}
$$

and

$$
\mathbf{T}_{3}-\mathbf{T}_{3} \mathbf{T}_{2}^{2}=\mathbf{S}\left(\begin{array}{cc}
\mathbf{0} & \mathbf{0} \\
\mathbf{0} & \mathbf{E}
\end{array}\right) \mathbf{S}^{-1}, \quad \mathbf{T}_{3} \mathbf{T}_{1}=\mathbf{S}\left(\begin{array}{cc}
\mathbf{0} & \mathbf{0} \\
\mathbf{0} & \mathbf{E K}
\end{array}\right) \mathbf{S}^{-1}
$$

the

Substituting these equalities in (2.64) leads to the formula (2.30) which is desired result. So the proof is complete.

In case when $c_{4}=0$, we get the following corollary.

Corollary 2.3. Let $c_{1}, c_{2}, c_{3} \in \mathbb{C}^{*}, \mathbf{T}_{1}, \mathbf{T}_{2}$, and $\mathbf{T}_{3} \in \mathcal{M}_{n}$ be nonzero tripotent matrices such that $\mathbf{T}_{1}^{2} \mathbf{T}_{2}-\mathbf{T}_{2}^{2} \mathbf{T}_{1}=\mathbf{T}_{2}^{2} \mathbf{T}_{3}+\mathbf{T}_{3}^{2} \mathbf{T}_{2}=\mathbf{T}_{1}^{2} \mathbf{T}_{3}-\mathbf{T}_{3}^{2} \mathbf{T}_{1}=\mathbf{0}$.

(i) If $\mathbf{T}_{1}$ is nonsingular, $c_{1}+c_{3} \neq 0$, and $c_{1}+c_{2} \neq 0$, then

$$
\begin{aligned}
& \left(c_{1}+c_{2}\right)\left(c_{1}+c_{3}\right)\left[c_{1} \mathbf{T}_{1}+c_{2} \mathbf{T}_{2}+c_{3} \mathbf{T}_{3}\right]^{-1} \\
& =\left(c_{1}+c_{3}\right) \mathbf{T}_{1} \mathbf{T}_{2}^{2}+\left(c_{1}+c_{2}\right)\left[c_{1}^{-1}\left(c_{1}+c_{3}\right)\left(\mathbf{T}_{1}-\mathbf{T}_{1} \mathbf{T}_{2}^{2}-\mathbf{T}_{1} \mathbf{T}_{3}^{2}\right)+\mathbf{T}_{1} \mathbf{T}_{3}^{2}\right],
\end{aligned}
$$

(ii) If $\mathbf{T}_{2}$ is nonsingular, $c_{1}+c_{2} \neq 0$, and $c_{2}-c_{3} \neq 0$, then

$$
\begin{aligned}
& \left(c_{1}+c_{2}\right)\left(c_{2}-c_{3}\right)\left[c_{1} \mathbf{T}_{1}+c_{2} \mathbf{T}_{2}+c_{3} \mathbf{T}_{3}\right]^{-1} \\
& =\left(c_{1}+c_{2}\right) \mathbf{T}_{2} \mathbf{T}_{3}^{2}+\left(c_{2}-c_{3}\right)\left[c_{2}^{-1}\left(c_{1}+c_{2}\right)\left(\mathbf{T}_{2}-\mathbf{T}_{2} \mathbf{T}_{3}^{2}-\mathbf{T}_{2} \mathbf{T}_{1}^{2}\right)+\mathbf{T}_{2} \mathbf{T}_{1}^{2}\right],
\end{aligned}
$$

(iii) If $\mathbf{T}_{3}$ is nonsingular, $c_{1}+c_{3} \neq 0$, and $c_{2}-c_{3} \neq 0$, then

$$
\begin{aligned}
& \left(c_{1}+c_{3}\right)\left(c_{3}-c_{2}\right)\left(c_{1} \mathbf{T}_{1}+c_{2} \mathbf{T}_{2}+c_{3} \mathbf{T}_{3}\right)^{-1} \\
& \quad=\left(c_{1}+c_{3}\right) \mathbf{T}_{3} \mathbf{T}_{2}^{2}+\left(c_{3}-c_{2}\right) c_{3}{ }^{-1}\left[\left(c_{1}+c_{3}\right)\left(\mathbf{T}_{3}-\mathbf{T}_{3} \mathbf{T}_{2}{ }^{2}\right)-c_{1} \mathbf{T}_{3} \mathbf{T}_{1}{ }^{2}\right] .
\end{aligned}
$$

Next theorem shows that the nonsingularity of

$$
c_{1} \mathbf{T}_{1}+c_{2} \mathbf{T}_{2}+c_{3} \mathbf{T}_{3}-c_{4}\left(\mathbf{T}_{1} \mathbf{T}_{2}+\mathbf{T}_{3} \mathbf{T}_{1}+\mathbf{T}_{2} \mathbf{T}_{3}\right)
$$

is also related to the nonsingularity of a combination of $\left(\mathbf{T}_{2}^{2}+\mathbf{T}_{3}^{2}\right) \mathbf{T}_{1},\left(\mathbf{T}_{1}^{2}+\mathbf{T}_{3}^{2}\right) \mathbf{T}_{2}$ and $\left(\mathbf{T}_{1}^{2}+\mathbf{T}_{2}^{2}\right) \mathbf{T}_{3}$ or $\mathbf{T}_{1}\left(\mathbf{T}_{2}^{2}+\mathbf{T}_{3}^{2}\right), \mathbf{T}_{2}\left(\mathbf{T}_{1}^{2}+\mathbf{T}_{3}^{2}\right)$ and $\mathbf{T}_{3}\left(\mathbf{T}_{1}^{2}+\mathbf{T}_{2}^{2}\right)$.

Theorem 2.8. Let $c_{1}, c_{2}, c_{3} \in \mathbb{C}^{*}, c_{4} \in \mathbb{C}$, and $\mathbf{T}_{1}, \mathbf{T}_{2}, \mathbf{T}_{3} \in \mathcal{M}_{n}$ be tripotent matrices. The following statements are equivalent:

(i) $c_{1}\left(\mathbf{T}_{2}^{2}+\mathbf{T}_{3}^{2}\right) \mathbf{T}_{1}+c_{2}\left(\mathbf{T}_{1}^{2}+\mathbf{T}_{3}^{2}\right) \mathbf{T}_{2}+c_{3}\left(\mathbf{T}_{1}^{2}+\mathbf{T}_{2}^{2}\right) \mathbf{T}_{3}-c_{4}\left(\left(\mathbf{T}_{2}^{2}+\mathbf{T}_{3}^{2}\right) \mathbf{T}_{1} \mathbf{T}_{2}\right.$ $\left.+\left(\mathbf{T}_{1}^{2}+\mathbf{T}_{2}^{2}\right) \mathbf{T}_{3} \mathbf{T}_{1}+\left(\mathbf{T}_{1}^{2}+\mathbf{T}_{3}^{2}\right) \mathbf{T}_{2} \mathbf{T}_{3}\right)$ is nonsingular. 
(ii) $c_{1} \mathbf{T}_{1}\left(\mathbf{T}_{2}^{2}+\mathbf{T}_{3}^{2}\right)+c_{2} \mathbf{T}_{2}\left(\mathbf{T}_{1}^{2}+\mathbf{T}_{3}^{2}\right)+c_{3} \mathbf{T}_{3}\left(\mathbf{T}_{1}^{2}+\mathbf{T}_{2}^{2}\right)-c_{4}\left(\mathbf{T}_{3} \mathbf{T}_{1}\left(\mathbf{T}_{2}^{2}+\mathbf{T}_{3}^{2}\right)\right.$ $\left.+\mathbf{T}_{2} \mathbf{T}_{3}\left(\mathbf{T}_{1}^{2}+\mathbf{T}_{2}^{2}\right)+\mathbf{T}_{1} \mathbf{T}_{2}\left(\mathbf{T}_{1}^{2}+\mathbf{T}_{3}^{2}\right)\right)$ is nonsingular.

(iii) $c_{1} \mathbf{T}_{1}+c_{2} \mathbf{T}_{2}+c_{3} \mathbf{T}_{3}-c_{4}\left(\mathbf{T}_{1} \mathbf{T}_{2}+\mathbf{T}_{3} \mathbf{T}_{1}+\mathbf{T}_{2} \mathbf{T}_{3}\right)$ and $\mathbf{T}_{1}^{2}+\mathbf{T}_{2}^{2}+\mathbf{T}_{3}^{2}-\mathbf{I}_{n}$ are nonsingular.

The proof of this theorem is followed immediately from the equalities

$$
\begin{aligned}
\left(\mathbf{T}_{1}^{2}+\right. & \left.\mathbf{T}_{2}^{2}+\mathbf{T}_{3}^{2}-\mathbf{I}_{n}\right)\left[c_{1} \mathbf{T}_{1}+c_{2} \mathbf{T}_{2}+c_{3} \mathbf{T}_{3}-c_{4}\left(\mathbf{T}_{1} \mathbf{T}_{2}+\mathbf{T}_{2} \mathbf{T}_{3}+\mathbf{T}_{3} \mathbf{T}_{1}\right)\right] \\
= & c_{1}\left(\mathbf{T}_{2}^{2}+\mathbf{T}_{3}^{2}\right) \mathbf{T}_{1}+c_{2}\left(\mathbf{T}_{3}^{2}+\mathbf{T}_{1}^{2}\right) \mathbf{T}_{2}+c_{3}\left(\mathbf{T}_{1}^{2}+\mathbf{T}_{2}^{2}\right) \mathbf{T}_{3} \\
& -c_{4}\left[\left(\mathbf{T}_{2}^{2}+\mathbf{T}_{3}^{2}\right) \mathbf{T}_{1} \mathbf{T}_{2}+\left(\mathbf{T}_{3}^{2}+\mathbf{T}_{1}^{2}\right) \mathbf{T}_{2} \mathbf{T}_{3}+\left(\mathbf{T}_{1}^{2}+\mathbf{T}_{2}^{2}\right) \mathbf{T}_{3} \mathbf{T}_{1}\right]
\end{aligned}
$$

and

$$
\begin{aligned}
{\left[c_{1} \mathbf{T}_{1}+c_{2} \mathbf{T}_{2}+c_{3} \mathbf{T}_{3}-c_{4}\left(\mathbf{T}_{1} \mathbf{T}_{2}+\mathbf{T}_{2} \mathbf{T}_{3}+\mathbf{T}_{3} \mathbf{T}_{1}\right)\right]\left(\mathbf{T}_{1}^{2}+\mathbf{T}_{2}^{2}+\mathbf{T}_{3}^{2}-\mathbf{I}_{n}\right) } \\
=c_{1} \mathbf{T}_{1}\left(\mathbf{T}_{2}^{2}+\mathbf{T}_{3}^{2}\right)+c_{2} \mathbf{T}_{2}\left(\mathbf{T}_{3}^{2}+\mathbf{T}_{1}^{2}\right)+c_{3} \mathbf{T}_{3}\left(\mathbf{T}_{1}^{2}+\mathbf{T}_{2}^{2}\right) \\
\quad-c_{4}\left[\mathbf{T}_{1} \mathbf{T}_{2}\left(\mathbf{T}_{1}^{2}+\mathbf{T}_{3}^{2}\right)+\mathbf{T}_{2} \mathbf{T}_{3}\left(\mathbf{T}_{1}^{2}+\mathbf{T}_{2}^{2}\right)+\mathbf{T}_{3} \mathbf{T}_{1}\left(\mathbf{T}_{2}^{2}+\mathbf{T}_{3}^{2}\right)\right] .
\end{aligned}
$$

Observe that setting $c_{4}=0$ in the last result, we get a characterization of the nonsingularity of a linear combination of three tripotent matrices without any further restriction on these matrices.

\section{References}

[1] J.K. Baksalary and O.M. Baksalary, Nonsingularity of linear combinations of idempotent matrices, Linear Algebra Appl. 388 (2004), pp. 25-29.

[2] J.K. Baksalary, O.M. Baksalary, and H. Özdemir, A note on linear combination of commuting tripotent matrices, Linear Algebra Appl. 388 (2004) 45-51.

[3] A. Ben-Israel and T.N.E. Greville, Generalized Inverses: Theory and Applications, 2nd ed, CMS Books in Mathematics, Springer-Verlag, New York, 2003.

[4] J. Benítez, X. Liu, and T. Zhu, Nonsingularity and group invertibility of linear combination of two k-potent matrices, Linear Multilinear Alg. 58 (2010), pp. 1023-1035.

[5] J. Benítez and N. Thome, k-Group periodic matrices, SIAM. J. Matrix Anal. Appl. 28 (2006), pp. 9-25.

[6] J. Groß and G. Trenkler, Nonsingularity of the difference of two oblique projectors, SIAM J. Matrix Anal. Appl. 21 (1999), pp. 390-395.

[7] R.A. Horn and C.R. Johnson, Matrix Analysis. Cambridge University Press, Cambridge, UK, 1985.

[8] J.J. Koliha and V. Rakočević, The nullity and rank of linear combinations of idempotent matrices, Linear Algebra Appl. 418 (2006), pp. 11-14.

[9] J.J. Koliha, V. Rakočević, and I. Straškraba, The difference and sum of projectors, Linear Algebra Appl. 388 (2004), pp. 279-288.

[10] X. Liu, S. Wu, and J. Benítez, On nonsingularity of combinations of two group invertible matrices and two tripotent matrices, Linear Multilinear Alg. iFirst (2011), pp. 1-9.

[11] X. Liu, L. Wu, and J. Benítez, On The Group Inverse Of Linear Combinations Of Two Group Invertible Matrices, Electron. J. Linear Algebra 22 (2011), pp. 490-503.

[12] C.D. Meyer, Matrix Analysis and Applied Linear Algebra. SIAM, Philadelphia, 2000. 
mitr315 [13] S.K. Mitra. On group inverses and the sharp order, Linear Algebra Appl. 92 (1987), pp. 17-37.

[14] S.K. Mitra, P. Bhimasankaram, and S.B. Malik Matrix Partial Orders, Shorted Operators and Applications. World Scientific.

[15] M. Sarduvan and H. Özdemir, On nonsingularity of linear combinations of tripotent matrices, Acta Universitatis Apulensis 25 (2011), pp. 159-164.

Zus2 [16] K. Zuo, Nonsingularity of the difference and the sum of two idempotent matrices, Linear Algebra Appl. 433 (2010), pp. 476-482.

[17] K. Zuo and T. Xie, Nonsingularity of combinations of idempotent matrices, J. Math. (PRC) 29 (2009), pp. 285-288.

Zhan 325 [18] F. Zhang, Matrix Theory. Springer Verlag New York inc., New York, 1999. 